Review Article

\title{
A Review of the Botany, Traditional Use, Phytochemistry, Analytical Methods, Pharmacological Effects, and Toxicity of Angelicae Pubescentis Radix
}

\author{
Liu Yang $\mathbb{D}^{1},{ }^{1}$ Ajiao Hou, ${ }^{1}$ Song Wang, ${ }^{1}$ Jiaxu Zhang, ${ }^{1}$ Wenjing Man, ${ }^{1}$ Xinyue Guo, \\ Bingyou Yang $\mathbb{D}^{1},{ }^{1}$ Hai Jiang $\mathbb{D},{ }^{1}$ Haixue Kuang $\mathbb{D}^{1},{ }^{1}$ and Qiuhong Wang $\mathbb{D}^{1,2}$ \\ ${ }^{1}$ Key Laboratory of Chinese Materia Medica, Heilongjiang University of Chinese Medicine, Ministry of Education, \\ Harbin 150040, China \\ ${ }^{2}$ School of Traditional Chinese Medicine, Guangdong Pharmaceutical University, Guangzhou 528458, China
}

Correspondence should be addressed to Hai Jiang; jianghai_777@126.com, Haixue Kuang; hxkuang56@163.com, and Qiuhong Wang; qhwang668@sina.com

Received 2 May 2020; Revised 17 June 2020; Accepted 27 June 2020; Published 3 August 2020

Academic Editor: Simona Martinotti

Copyright $\odot 2020$ Liu Yang et al. This is an open access article distributed under the Creative Commons Attribution License, which permits unrestricted use, distribution, and reproduction in any medium, provided the original work is properly cited.

\begin{abstract}
Angelicae Pubescentis Radix (AP), as a traditional Chinese medicine (TCM), has been used for thousands of years in China. In this paper, the botany, traditional use, phytochemistry, analytical methods, quality control, pharmacological effects, and toxicity of AP were reviewed. It can provide a reference for the further research and lay a foundation for the rational clinical application of AP. The relevant information on AP was collected from scientific databases (such as Baidu Scholar, CNKI, Google Scholar, PubMed, Science Direct, Web of Science, and SciFinder Scholar), Chinese herbal classics, Chinese Pharmacopoeia, PhD and MSc dissertations, and so on. The components which have been isolated and identified in AP include coumarins, volatile oils, organic acids, terpenes, polysaccharides, flavonoids, sterols, and trace elements. Most of them were analyzed by HPLC and GC. A pharmacological study shows that the AP has extensive pharmacological effects, including anti-inflammatory, antirheumatism, sedative and hypnotic, neuroprotection, antioxidation, antitumor, and allergy, and it is widely used in the treatment of the rheumatoid arthritis, knee osteoarthritis, lumbar disc, ankylosing spondylitis, headaches, stroke hemiplegia, Alzheimer's, and arrhythmia. AP is a valuable natural medicinal plant. So far, significant advances have been made in phytochemistry and pharmacology. Some traditional uses have been demonstrated by modern pharmacology. However, the chemical components and pharmacological effects of AP are complex and varied, and there are different standards for the evaluation of its quality and efficacy. The mechanism of action, the structure-activity relationship among the components, and the potential synergistic and antagonistic effects remain to be studied. At the same time, there are few studies on the specific compounds related to its pharmacodynamics. In order to better develop and utilize AP, we should establish a more reasonable, reliable, and accurate quality control standard and focus on the study of bioactive constituents and the demonstration of their mechanism of action.
\end{abstract}

\section{Introduction}

Angelicae Pubescentis Radix (AP) is derived from the dry root of Angelica pubescens Maxim f. biserrata Shan et Yuan, a plant in the Apiaceae family. AP was first published in Sheng Nong's herbal classic, which is spicy, bitter, and mild in nature and enters the kidney meridian and bladder meridian exerting the remedial effect [1]. AP was recorded and summarized by each edition of the Chinese Pharmacopoeia, with the functions of removing wind and dehumidification, relieving pain in paralysis, and so on. AP was often used to treat rheumatism and headaches caused by dampness and cold [2].

A phytochemical study shows that the main active components of AP include volatile oil, coumarin, organic acids, terpenes, polysaccharides, sterols, and other compounds. And, the pharmacological studies show that AP has anti-inflammatory, antirheumatic, sedative, hypnotic, 
neuroprotective, antioxidant, antitumor, antiallergic, and other effects, which may be closely related to its complex chemical components [3-10]. In clinical application, AP is often used in combination with other TCMs, which has the functions of dispelling wind and dehumidification, promoting blood circulation and relieving pain, relaxing muscles and activating collaterals, tonifying the liver and kidney, and strengthening tendons and bones [11-15], such as Duhuo-Jisheng Wan, Tianhe Zhuifeng Gao, and Tianma Wan. It is usually used to treat arthritis, lumbago, and cephalgia caused by cold and dampness, blocking collaterals of blood stasis, and deficiency of qi and blood [16-18].

$\mathrm{AP}$, as a famous TCM, has been widely used in China for thousands of years. However, there was no in-depth study on the material basis, target, and mechanism of action of AP. There was no optimal processing technique for AP. And, at present, the toxic components and the related mechanism also have not been clarified. In this study, a total of 551 articles were identified from the database in this study, most of which were excluded due to no mention of botany, traditional use, phytochemistry, pharmacological effects of AP, or duplication. 129 references are included in this review. Compared with other reviews, this study provides a more comprehensive overview of botany, traditional uses, phytochemistry, pharmacological effects, and toxicology, with additional reviews of analytical methods, quality control, and processing. It also emphasizes its possible future development direction, which lays a foundation for a comprehensive understanding of AP, further research and development of new drugs, and expansion of its application in clinical and world markets.

\section{Botany}

The Chinese Pharmacopoeia (2015 edition) records AP derived from the dry root of Angelica pubescens Maxim, f. biserrata Shan et Yuan. In early spring, when the seedlings are just germinating, or in late autumn, when the stems and leaves are withered, the soil is digged, the fibrous roots and sediments are removed, and then the roots are baked to half dry, piled up for 2 to 3 days, putting to soft , and then baking to dry. DH is mainly distributed in Sichuan and Hubei provinces of China [2]. Native plants grow on the dank hillside, grass, or sparse thickets, like in a cool and humid climate, and are cold resistant, and most native plants grow in the altitude of 1200 2000 meters of the cold highland area. The plant is suitable to grow in fertile, loose alkaline soil, yellow sand soil, or black oil soil but not suitable to grow in the shallow soil, water, and clay soil [19]. There are a variety of invention patents for AP cultivation, planting, and harvesting methods, such as an AP cultivation method (CN. 201911138333) [20], an AP cultivation greenhouse (CN. 201811562359) [21], a method for cultivating high-quality AP (CN. 201810917290) [22], an AP cultivation method for high quality and high yield (CN. 201811053800), and also [23].

The roots of AP are thick and short, slightly cylindrical, $1.5-4 \mathrm{~cm}$ in length, and $1.5-3.5 \mathrm{~cm}$ in diameter. The lower roots have several curved branches, $12-30 \mathrm{~cm}$ in length, and $0.5-1.5 \mathrm{~cm}$ in diameter. The root surface is rough, graybrown, with irregular longitudinal wrinkles and transverse cracks, and has many transverse long lenticels and fine roots [24]. The root head has a ring stripe and polytropic ring petiole mark, and the hollow stem mark is in the middle. Texture is hard, cross section is sallow white, and the cambium is ring brown. The leather part has a brown oil point (tubing), and the wood part is yellow brown. The transverse section of the root has a large curved section and oil spots. AP has a special flavor. It tastes bitter and hot and even can make a tongue numb. AP with thick, oily, and strong aroma are for the best [25].

\section{Traditional Applications}

Sheng Nong's herbal classic (Han dynasty, 947-950) records that AP can treat diseases caused by wind and cold, pain caused by bumping of knife wounds, epilepsy, female uterine fibroid hernia, etc. It is recorded in the Supplementary Records of Famous Physicians (the end of Han dynasty, 947-950) that AP can cure all kinds of limb joint pain caused by wind and evil spirits, whether it is a new disease or a long illness. Medicinal Theory (Tang dynasty, 923-936) records that AP can treat the pain caused by wind, cold and wet, qi disorder, itchy skin, pain caused by limb spasm, pain caused by fatigue, and tooth pain. According to Medical Origins (Jin dynasty, 1186), AP can remove moisture and treat headaches and dizziness. Wang Haogu (Yuan dynasty, 1271-1368) shows that AP could cure the pain in the waist and leg. According to the book "Yeyan" (Ming dynasty, 1368-1644), AP can be used for sweating, treatment of flank pain, and head and facial pain. Bencao Tongxuan (Ming dynasty, 1368-1644) records that AP is used for the treatment of speechlessness, stiff hands and feet, mouth and eyes skewness, swollen eyes, and skin itching. Bencaozheng (Ming dynasty, 1368-1644) records that AP can cure rheumatism, foot pain, itching, and stiff limbs. According to Modern Practical Chinese Medicine, AP can induce sweating, promote urine discharge, and eliminate edema.

Clinically, AP is often used in combination with other TCMs. The dried root of the TCMs commonly used in combination with AP are as follows:

(1) AP compatibility with Paeoniae Radix Alba, which has the functions of tonifying liver and dispelling wind [11].

(2) AP compatibility with Dictamni Cortex, which has the functions of dispelling wind and dehumidification [11].

(3) AP compatibility with Asari Radix et Rhizoma, which has the functions of dispelling wind and dehumidification, dredging paralysis, and relieving pain [11].

(4) AP compatibility with Taxilli Herba, which has the functions of dispelling wind and dehumidification, tonifying kidney, and dredging paralysis [12].

(5) AP compatibility with Rehmanniae Radix, which has the functions of tonifying liver, kidney, and blood, strengthening tendons and bones, tonifying yin, and promoting fluid production [12]. 
(6) AP compatibility with Angelicae sinensis Radix, which has the functions of tonifying blood and dispelling wind [12].

(7) AP compatibility with Typhonii Rhizoma, which has the functions of dispelling wind, dehumidification, relaxing muscles and activating collaterals, and relieving pain [12].

(8) AP compatibility with Ligustici Rhizoma et Radix, which has the functions of dispelling wind and dehumidification, dissipating cold, and relieving pain [12].

(9) AP compatibility with Schizonepetae Herba, which has the functions of dehumidification and relieving spasm, relaxing muscles, and activating collaterals [12].

(10) AP compatibility with Ephedrae Herba, which has the functions of dispelling wind and removing fever, dehumidification, and relieving pain [12].

(11) AP compatibility with Notopterygh Rhizoma et Radix, which has the functions of dispelling wind and dehumidification, dredging paralysis, and relieving pain $[13,14]$.

(12) AP compatibility with Gentianae Macrophyllae Radix, which has the functions of dispelling wind and dehumidification, dredging paralysis, and relieving pain [15].

(13) AP compatibility with Phellodendri Amurensis Cortex, which has the functions of dehumidification and antipyretic [15].

The commonly used clinical prescriptions include Duhuo-Jisheng decoction [26], Duhuo-Cangzhu decoction, Duhuo decoction [27], Duhuo-Xixin decoction [28], and Duhuo pulvis [29]. Among them, Duhuo-Jisheng decoction is most widely used in clinical practice, which has good effects on rheumatoid arthritis, rheumatoid arthritis, knee osteoarthritis, lumbar disc herniation, headache, stroke, hemiplegia, and other diseases. The dosage forms involved include decoction, liquor, tablet, capsule, pill, aerosol, liniment, powder, paste, etc. [2] (as shown in Table 1).

In addition, it is also used in health care, beauty, and other fields. As early as in Sheng Nong's herbal classic, it is recorded that "taking AP for a long time has weight loss and antiaging effects." The AP wine is recorded in Qianjinfang, "Medicinal tea for all kinds of diseases" records the AP tea. Peaceful Holy Benevolent Prescriptions records the APGinseng Radix et Rhizoma wine. General Records of Holy Universal Relief records the AP-Angelicae Sinensis Radix wine. In recent years, there are several patented inventions in health care and cosmetics. A kind of AP internal injuries fever health tea (CN. 2012103901941) [30], a kind of beauty dispelling wet medicinal wine (CN. 201710879392) [31], a beauty mask cream (201710782640.6), etc. [32].

\section{Chemical Component}

4.1. Coumarins. Coumarin compounds is the general name of o-hydroxycinnamic acid lactones with the basic skeleton of benzo $\alpha$-pyranone, which are one of the main components of AP [33]. In addition, Chinese Pharmacopoeia (2015 edition) also uses two coumarin components, osthol and columbianadin, as the quality control indexes of AP.

At present, more than 120 kinds of coumarins have been isolated from various varieties of AP, including furanocoumarins [34], pyranocoumarins [35], dicoumarins [35], simple coumarins [36-41], and individual coumarin glycosides [42]. The components of coumarins isolated from AP are listed in Table 2, and the structures are shown in Figure 1.

4.2. Volatile Oils. Volatile oil is a group name of volatile oillike compounds which has aromatic smell and can be distilled with water vapor without being miscible with water [46]. The volatile oil is a kind of mixture with relatively complex compounds. There are a large number of volatile oil components in AP, mainly including terpenoids, aromatic compounds, and aliphatic compounds [44,47-60], which is shown in Table 3. Gao et al. analyzed the volatile oil compounds from the water extract of AP and Heracleum candicans Wall ex Dc dried roots by GC/MS and identified 32 and 45 compounds, respectively [62]. Zhang et al. extracted the volatile oil compounds from the water extract of Heracleum hemsleyanum Diels dried roots and identified 53 compounds [63]. Wang et al. extracted and compared the volatile oil compounds of the dried roots and rhizomes of AP and Angelica dahurica (Fisch) Benth et Hook, and 90 compounds were detected and 52 were identified from AP; 57 compounds were detected from the dried roots and rhizomes of Angelica dahurica (Fisch) Benth et Hook and 37 were identified [64]. Yang et al. used steam distillation to extract the volatile oil from AP and detected 229 chromatography peaks by GC-MS and identified 88 compounds [65]. Zhang et al. extracted the volatile oil from the dried roots of Heracleum lanatum Michx by different extraction methods and identified a total of 59 compounds [61]. It has been reported that $40 \%$ of the small molecular compounds will change in the process of extraction and detection. Therefore, in this section, only list of the separated compounds from volatile oil of AP is discussed [66]. And, the compounds isolated and identified by these research studies are different, and even the same compounds have different content proportions in different varieties of AP, which may be related to the different species, climatic and environment of the cultivation area, soil conditions, processing methods of the production area, extraction facilities, and extraction conditions.

4.3. Organic Acids. Organic acid refers to a kind of acid organic compounds containing the carboxyl group, which has a variety of pharmacological effects such as antibacterial, antiviral, antidepression, antidiabetes, antitumor [66], antiinflammatory [67], antitussive, expectorant [68], and antioxidant [69]. With the first separation of 3-O-trans-coumaroylquinic acid, 3-O-trans-feruloylquinic acid, and other compounds from the methanol extract of the dried roots of AP by Yang et al., some scholars had developed great interest 
TABLE 1: The prescription composition, dosage form, and efficacy of AP.

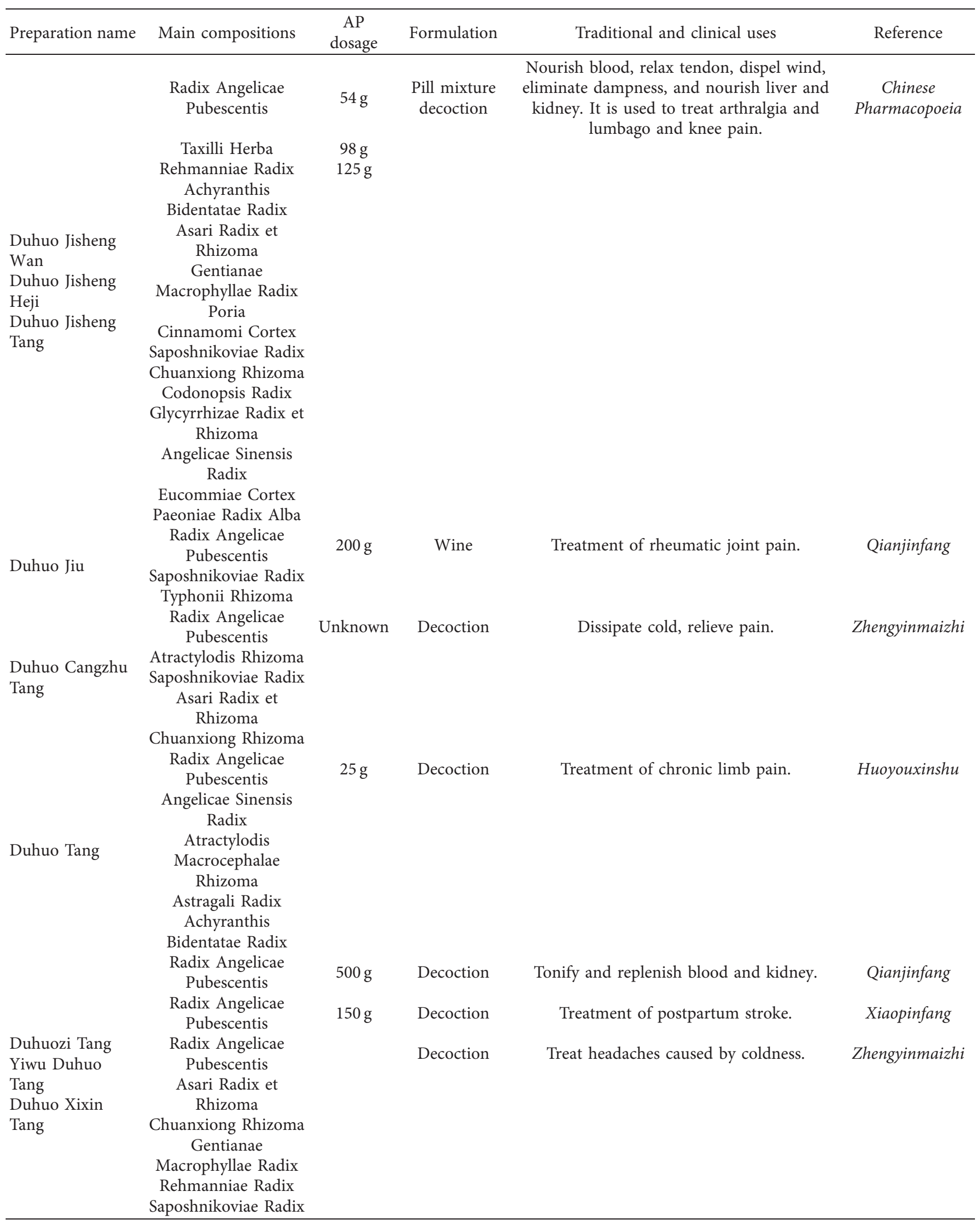


TABle 1: Continued.

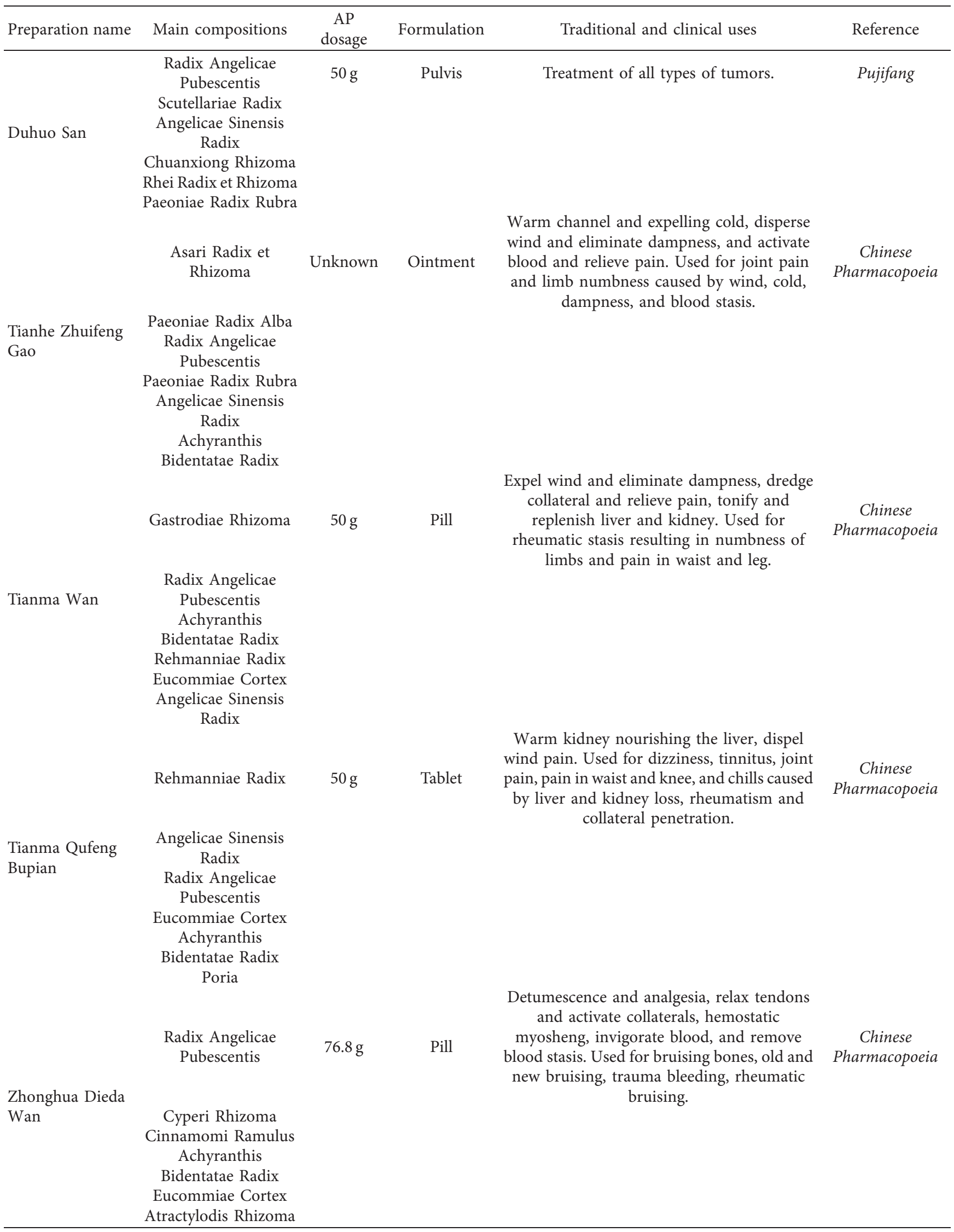


TABle 1: Continued.

\begin{tabular}{|c|c|c|c|c|c|}
\hline Preparation name & Main compositions & $\begin{array}{c}\text { AP } \\
\text { dosage }\end{array}$ & Formulation & Traditional and clinical uses & Reference \\
\hline \multirow{2}{*}{$\begin{array}{l}\text { Zhengtian Wan } \\
\text { Zhengtian } \\
\text { Jiaonang }\end{array}$} & $\begin{array}{c}\text { Angelicae Sinensis } \\
\text { Radix }\end{array}$ & $102 \mathrm{~g}$ & Pill, capsule & $\begin{array}{l}\text { Expel wind and activate blood, nourish blood } \\
\text { and tonic liver, dredge collateral and relieve } \\
\text { pain. It is used for migraines, tension } \\
\text { headache, nerve headache, cervical } \\
\text { spondylosis type headache, and premenstrual } \\
\text { headache caused by external wind evil, blood } \\
\text { stasis, blood loss of nutrition, hyperactivity of } \\
\text { liver yang. }\end{array}$ & $\begin{array}{c}\text { Chinese } \\
\text { Pharmacopoeia }\end{array}$ \\
\hline & $\begin{array}{l}\text { Paeoniae Radix Alba } \\
\text { Saposhnikoviae Radix } \\
\text { Asari Radix et } \\
\text { Rhizoma } \\
\text { Radix Angelicae } \\
\text { Pubescentis } \\
\text { Ephedrae Herba }\end{array}$ & & & & \\
\hline \multirow{2}{*}{$\begin{array}{l}\text { Zhuanggu } \\
\text { Guanjie Wan }\end{array}$} & Cibotii Rhizoma & Unknown & Pill & $\begin{array}{l}\text { Tonify the liver and kidney, nourish blood } \\
\text { and promote blood circulation, relax tendons } \\
\text { and collaterals, regulate qi and relieve pain. } \\
\text { Used for the treatment of arthritis, lumbar } \\
\text { muscle strain, joint swelling, pain, numbness, } \\
\text { limited movement. }\end{array}$ & $\begin{array}{c}\text { Chinese } \\
\text { Pharmacopoeia }\end{array}$ \\
\hline & $\begin{array}{c}\text { Radix Angelicae } \\
\text { Pubescentis } \\
\text { Dipsaci Radix } \\
\text { Taxilli Herba } \\
\text { Rehmanniae Radix } \\
\text { Psoraleae Frsuctus }\end{array}$ & & & & \\
\hline \multirow[b]{2}{*}{$\begin{array}{l}\text { Wangbi Pian } \\
\text { Wangbi Keli }\end{array}$} & Rehmanniae Radix & Unknown & Tablet & $\begin{array}{l}\text { Tonify and replenish liver and kidney, } \\
\text { strengthen tendons and bones. Disperse wind } \\
\text { and dehumidification, dredge collateral and } \\
\text { relax tendon. Used to treat rheumatoid } \\
\text { arthritis. }\end{array}$ & $\begin{array}{c}\text { Chinese } \\
\text { Pharmacopoeia }\end{array}$ \\
\hline & $\begin{array}{c}\text { Dipsaci Radix } \\
\text { Radix Angelicae } \\
\text { Pubescentis } \\
\text { Saposhnikoviae Radix } \\
\text { Rehmanniae Radix } \\
\text { Clematidis Radix et } \\
\text { Rhizoma }\end{array}$ & & & & \\
\hline \multirow{7}{*}{ Guogong Jiu } & $\begin{array}{l}\text { Angelicae Sinensis } \\
\text { Radix }\end{array}$ & Unknown & Wine & $\begin{array}{l}\text { Disperse wind and dehumidification, relax } \\
\text { sinew and dredge collateral. Used for the } \\
\text { treatment of joint pain, adverse flexion and } \\
\text { extension, hand and foot numbness, pain in } \\
\text { waist and leg. It is also used for hemiplegia } \\
\text { and askew caused by imbalance between } \\
\text { channels and collaterals. }\end{array}$ & $\begin{array}{c}\text { Chinese } \\
\text { Pharmacopoeia }\end{array}$ \\
\hline & Achyranthis & & & & \\
\hline & Bidentatae Radix & & & & \\
\hline & Radix Angelicae & & & & \\
\hline & $\begin{array}{l}\text { Pubescentis } \\
\text { Paeoniae Radix Alba }\end{array}$ & & & & \\
\hline & Psoraleae Fructus & & & & \\
\hline & Saposhnikoviae Radix & & & & \\
\hline
\end{tabular}


TABle 1: Continued.

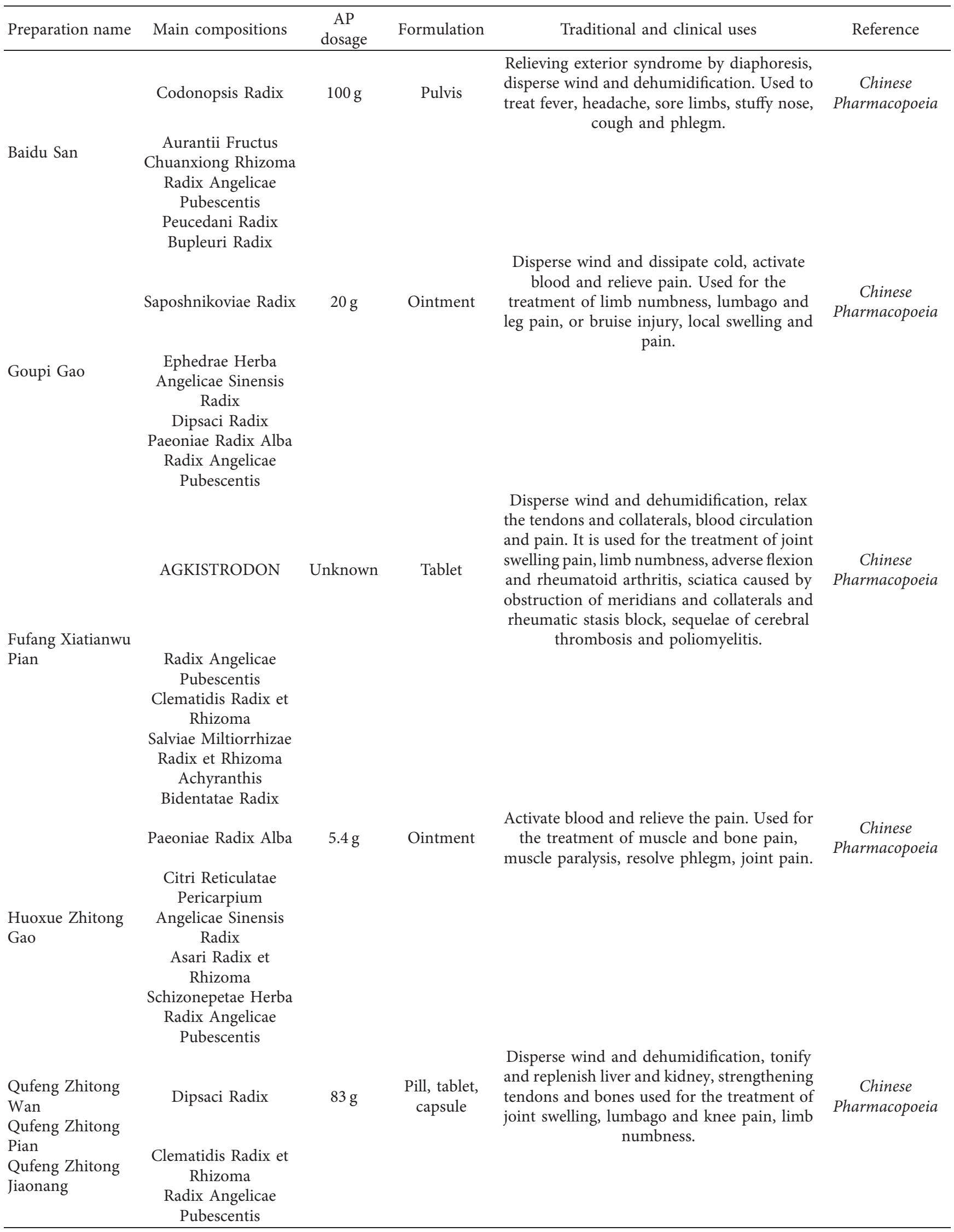


Table 1: Continued.

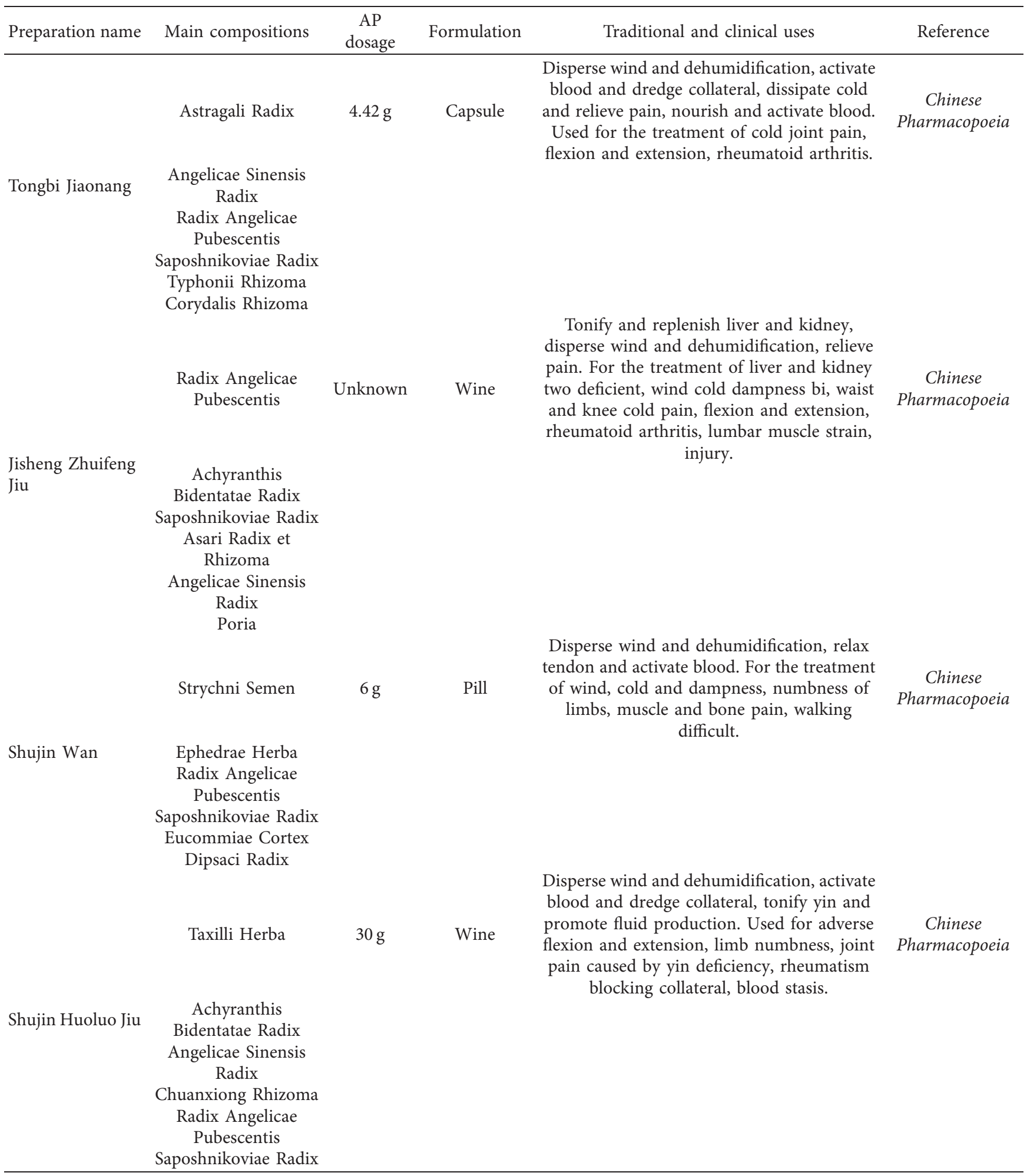


TABLE 1: Continued.

\begin{tabular}{|c|c|c|c|c|c|}
\hline Preparation name & Main compositions & $\begin{array}{c}\text { AP } \\
\text { dosage }\end{array}$ & Formulation & Traditional and clinical uses & Reference \\
\hline \multirow{8}{*}{$\begin{array}{l}\text { Shufeng } \\
\text { Dingtong Wan }\end{array}$} & Strychni Semen & \multirow[t]{8}{*}{$30 \mathrm{~g}$} & \multirow[t]{8}{*}{ Pill } & $\begin{array}{l}\text { Disperse wind and dissipate cold, activate } \\
\text { blood and relieve pain. Used for joint pain, } \\
\text { cold pain, stabbing pain, waist and leg pain } \\
\text { and limb numbness caused by wind, cold and } \\
\text { wet closure, and blood stasis. Local swelling } \\
\text { caused by a bruise. }\end{array}$ & \multirow[t]{2}{*}{$\begin{array}{c}\text { Chinese } \\
\text { Pharmacopoeia }\end{array}$} \\
\hline & Achyranthis & & & & \\
\hline & Bidentatae Radix & & & & \\
\hline & Saposhnikoviae Radix & & & & \\
\hline & Radix Angelicae & & & & \\
\hline & Pubescentis & & & & \\
\hline & Ephedrae Herba & & & & \\
\hline & Eucommiae cortex & & & & \\
\hline \multirow{8}{*}{$\begin{array}{l}\text { Yaobitong } \\
\text { Jiaonang }\end{array}$} & $\begin{array}{c}\text { Notoginseng Radix et } \\
\text { Rhizoma }\end{array}$ & \multirow[t]{8}{*}{ Unknown } & \multirow[t]{8}{*}{ Capsule } & $\begin{array}{c}\text { Activate blood and resolve stasis, dispel wind } \\
\text { and eliminate dampness, move qi and stop } \\
\text { pain. Used for lumbago caused by blood stasis } \\
\text { and qi stagnation. }\end{array}$ & $\begin{array}{c}\text { Chinese } \\
\text { Pharmacopoeia }\end{array}$ \\
\hline & Chuanxiong Rhizoma & & & & \\
\hline & Corydalis Rhizoma & & & & \\
\hline & Paeoniae Radix Alba & & & & \\
\hline & Achyranthis & & & & \\
\hline & Bidentatae Radix & & & & \\
\hline & Radix Angelicae & & & & \\
\hline & Pubescentis & & & & \\
\hline \multirow{7}{*}{$\begin{array}{l}\text { Shexiang Qutong } \\
\text { Qiwuji } \\
\text { Shexiang Qutong } \\
\text { Chaji }\end{array}$} & Moschus & \multirow[t]{7}{*}{$1 \mathrm{~g}$} & \multirow[t]{7}{*}{$\begin{array}{l}\text { Aerosol } \\
\text { liniment }\end{array}$} & $\begin{array}{l}\text { Activate blood and resolve stasis, relax sinew } \\
\text { and activate collateral, eliminate swelling and } \\
\text { relieve pain. Used for all kinds of fall injury, }\end{array}$ & \multirow[t]{7}{*}{$\begin{array}{c}\text { Chinese } \\
\text { Pharmacopoeia }\end{array}$} \\
\hline & & & & $\begin{array}{l}\text { Diood stasis sweilng pain, rneumatism stasis, } \\
\text { joint pain. }\end{array}$ & \\
\hline & Carthami Flos & & & & \\
\hline & Radix Angelicae & & & & \\
\hline & Pubescentis & & & & \\
\hline & Rehmanniae Radix & & & & \\
\hline & $\begin{array}{c}\text { Notoginseng Radix et } \\
\text { Rhizoma }\end{array}$ & & & & \\
\hline
\end{tabular}

in organic acids compounds in AP [70]. Up to now, the organic acid compounds isolated from AP are far more than these (as shown in Table 4 and Figure 2). We believe that with the progress of time and the development of science and technology, the organic acids in AP will be studied more thoroughly.

4.4. Polysaccharides. Polysaccharide compounds have many pharmacological effects, such as antitumor [72], immunomodulatory [73], hypoglycemic [74], hypolipidemic [75], antioxidant [76], and antiaging [77]. Studies have shown that polysaccharides can be obtained from the above-ground parts of AP: $6-\mathrm{O}-\beta$-D-apiofuranosyl-(1 $\longrightarrow 6)-\beta$-D-glucopyranosyl scopoletin, $7-\mathrm{O}-\beta$-D-apiofuranosyl- $(1 \longrightarrow 6)$ $\beta$-D-glucopyranosyl umbelliferone, 7 -O- $\beta$-D-glucopyranosyl umbelliferone, $3-\mathrm{O}-[\beta$-D-galactopyranose- $(1 \longrightarrow 2)$ $[\beta$-D-xylopyranose- $(1 \longrightarrow 4)]-\beta$-D-pyranoglucuronic acid]$28-\mathrm{O}-\beta$-D-pyranogluconoleanolic acid, 3-O-[ $\beta$-D-galactopyranose- $(1 \longrightarrow 2)-\beta$-D-pyranoglucuronic acid]-O$[\beta$-D-galactopyranose- $(1 \longrightarrow 2)-\beta$-D-pyranoglucuronic
acid]-28-O- $\beta$-D-pyran glucose ivy saponins, 3-O-[ $\beta$-Dgalactopyranose-(1 $\longrightarrow 2)-\beta$-D-pyranoglucuronic acid]-28O- $\beta$-D-pyranogluconoleanolic acid, 3-O-[ $\beta$-D-galactopyranose-(1 $\longrightarrow 2)-\beta$-D-pyranoglucuronic acid] oleanolic acid, 3-O-[ $\beta$-D-xylopyranose- $(1 \longrightarrow 4)-\beta$-D-pyranoglucuronic acid]-28-O- $\beta$-D-pyran galactose oleanolic acid, 3-O-[ $\beta$-Dxylopyranose- $(1 \longrightarrow 4)-\beta$-D-pyranoglucuronic acid]-28-O$\beta$-D-pyranogluconoleanolic acid, 3-O-[ $\beta$-D-xylopyranose$(1 \longrightarrow 4)-\beta$-D-pyranoglucuronic acid]-hederagenin, 3-O$[\beta$-D-xylopyranose- $(1 \longrightarrow 4)-\beta$-D-pyranoglucuronic acid]oleanolic acid, 3-O- $\beta$-D-pyranoglucuronic acid-28-O- $\beta$-Dpyranogluconoleanolic acid, and $3-\mathrm{O}-\beta-\mathrm{D}$-pyranoglucuronic acid-oleanolic acid $[9,42,48]$.

4.5. Others. There are also compounds like adenine riboside, adenosine, allantion, angesesquid $A$, angesesquid $B, 2,3,4,9$ cartrahyd-ro-1-H-pyridio[3,4,-b]indole-3-carboxylic acid 1, $\alpha$-caryophyllene, coclaurine, daucosterol, glyceride, glucose, liriodenine, 1-N-methylcoclaurine, oleanic acid, sucrose, $\beta$-sitosterol, uridine, diterpenic acid, diterpene alcohols, and 
TABLE 2: The coumarin compounds of AP.

\begin{tabular}{|c|c|c|}
\hline No. & Compounds & Reference \\
\hline 1 & Acaculetindimethylether & {$[43]$} \\
\hline 2 & Angelicae lactone aldehyde-6-formyl-7-methoxycoumarin & {$[43]$} \\
\hline 3 & Allimperatorin & {$[43]$} \\
\hline 4 & Angelicin & {$[43]$} \\
\hline 5 & Angelicone & {$[43]$} \\
\hline 6 & Angelidiol & {$[43]$} \\
\hline 7 & Angelin & {$[43]$} \\
\hline 8 & Angelitriol & {$[43]$} \\
\hline 9 & Angelmarin & {$[43]$} \\
\hline 10 & Angelol A & {$[43]$} \\
\hline 11 & Angelol B & [43] \\
\hline 12 & Angelol C & {$[43]$} \\
\hline 13 & Angelol D & [43] \\
\hline 14 & Angelol E & {$[43]$} \\
\hline 15 & Angelol F & {$[43]$} \\
\hline 16 & Angelol G & {$[43]$} \\
\hline 17 & Angelol H & {$[43]$} \\
\hline 18 & Angelol I & {$[43]$} \\
\hline 19 & Angelol J & {$[43]$} \\
\hline 20 & Angelol K & {$[43]$} \\
\hline 21 & Angelol L & {$[43]$} \\
\hline 22 & Angenomalin & {$[44]$} \\
\hline 23 & Angepubebisin & {$[43]$} \\
\hline 24 & Anhydrobyakangelin & [43] \\
\hline 25 & Anpubesol & [43] \\
\hline 26 & Apaensin & [43] \\
\hline 27 & Apiosylckimmin & {$[43]$} \\
\hline 28 & Apterin & {$[43]$} \\
\hline 29 & Bergapten & {$[43]$} \\
\hline 30 & Bergaptol & {$[43]$} \\
\hline 31 & Bisabolangelone & {$[45]$} \\
\hline 32 & Byakangelicin & {$[43]$} \\
\hline 33 & Byakangelicol & {$[43]$} \\
\hline 34 & Cnidilin & {$[43]$} \\
\hline 35 & Columbianetin & {$[43]$} \\
\hline 36 & Columbianetin & {$[43]$} \\
\hline 37 & Columbianetin acetate & {$[43]$} \\
\hline 38 & Columbianetin- $\beta$-D-glucopyranoside & {$[43]$} \\
\hline 39 & Columbianetin propionate & {$[45]$} \\
\hline 40 & Columbianadin & {$[43]$} \\
\hline 41 & Coumurrayin & {$[43]$} \\
\hline 42 & Dehydra-angelol A & [39] \\
\hline 43 & Dehydra-angelol B & [39] \\
\hline 44 & Dehydra-angelol C & {$[39]$} \\
\hline 45 & 2-Deoxymeranzin hydrate & {$[44]$} \\
\hline 46 & Dihydrocolumbianadin & {$[45]$} \\
\hline 47 & Ferulin & {$[41]$} \\
\hline 48 & 8-(3-Hydroxylsoval-croyl-5,7-dimethoxycoumarin) & {$[43]$} \\
\hline 49 & 5-(2-Hydroxy-3-methoxy-3-methylbutoxy-psoralen) & {$[43]$} \\
\hline 50 & Imperatorin & {$[43]$} \\
\hline 51 & Isoangenomalin & {$[45]$} \\
\hline 52 & Isoanglol & {$[43]$} \\
\hline 53 & Isobergapten & {$[43]$} \\
\hline 54 & Isoimperatorin & {$[43]$} \\
\hline 55 & Isooxypencedanine & {$[43]$} \\
\hline 56 & Isopimpinellin & {$[43]$} \\
\hline 57 & Isopsoralen & {$[43]$} \\
\hline 58 & Marmesinin & {$[43]$} \\
\hline 59 & Meranzin hydrate & {$[43]$} \\
\hline 60 & 7-Methoxy-8-sene-cloylcoumarin & [43] \\
\hline
\end{tabular}


TABle 2: Continued.

\begin{tabular}{lcc}
\hline No. & Compounds & Reference \\
\hline 61 & Neobyakangelicol & {$[43]$} \\
62 & Nodakenetin & {$[45]$} \\
63 & Nodakenin & {$[43]$} \\
64 & Osthenol & {$[43]$} \\
65 & Osthol & {$[43]$} \\
66 & Oxypeucedanin & {$[43]$} \\
67 & Oxypeucedania hydrate \\
68 & Pabulenol \\
69 & Peucedanol \\
70 & Psoralen \\
71 & Scopoletin \\
72 & sec-O-Acetyl-byakangelicin \\
73 & sec-O- $\beta$-D-Glucopy-ranosyl-(R)-byakangelicin \\
74 & tert-O- $\beta$-D-Glucopy-ranosyl-(R)-byakangelicin \\
75 & Ulopterol \\
76 & Umbelliferone \\
77 & Umbelliprenin \\
78 & Vaginidiol \\
79 & Xanthotoxin \\
\hline
\end{tabular}

other compounds and $\mathrm{Al}, \mathrm{As}, \mathrm{B}, \mathrm{Ba}, \mathrm{Ca}, \mathrm{Cd}, \mathrm{Co}, \mathrm{Cr}, \mathrm{Cs}, \mathrm{Cu}$, $\mathrm{Fe}, \mathrm{Ge}, \mathrm{Hg}, \mathrm{K}, \mathrm{Mg}, \mathrm{Mo}, \mathrm{Mn}, \mathrm{Na}, \mathrm{Ni}, \mathrm{P}, \mathrm{Pb}, \mathrm{Se}, \mathrm{Sr}, \mathrm{Zn}$, and other trace elements in AP [78].

In this section, the chemical constituents of AP are summarized, which is helpful to further study on the material basis of its efficacy and explore the principle of its prevention and treatment of diseases. And, it also can provide an effective scientific basis for the development of new drugs, the expansion of drug sources, the synthesis of lead compounds or new drugs, the adaptation to clinical use, and the expansion of its market application.

\section{Analysis Method and Quality Control}

There are many kinds of compounds in AP, and their analysis methods are various. And, volatile oil compounds are often detected by gas chromatography-tandem mass spectrometry (GC-MS) [56]. The authors in [79] used the colorimetry method to determine the content of total coumarins in AP. However, the sample preparation of this method is complex and time consuming. Subsequently, thin layer chromatography (TLC) was used to determine the content of columbianetin. Although this method is simple, it is not precise enough and is not ideal for the separation of multicomponent compounds [64]. Some scholars used highperformance liquid chromatography (HPLC) [80-83], highperformance liquid chromatography in tandem with diode array detector (HPLC-DAD) [84,85], high-performance liquid chromatography in tandem with ultraviolet detector (HPLC-UV) [86,87], ultraperformance liquid chromatography-photo diode array detection(UPLC-PDA) [88], and other methods to determine the flavonoids, coumarins, and other compounds with UV absorption in AP. The above methods can analyze the compounds in AP and have good separation effect and stability. However, these methods are not suitable for compounds with no UV absorption or only terminal absorption. There are also scholars who used ultrahigh-performance liquid phase tandem mass spectrometry (UPLC-MS/MS) to determine more than 40 chemical components such as coumarins and phenolic acids in AP. Hou et al. used ultrahigh-performance liquid phase tandem single quadrupole mass spectrometry (UPLC-QDA) to establish the fingerprint of AP, which not only analyzed the type and quantity of the whole compound of AP, but also made up for the deficiency that the above method could not detect compounds with no UV absorption. Wang et al. used high-performance liquid chromatography in tandem with fluorescence detection (HPLC-FD) to determine umbelliferones and scopoletins in AP. The results show that this method is easy to operate, economical, and practical [72]. Yang et al. used ultraperformance convergence chromatography (UPCC) and $\mathrm{CO}_{2}$ as the mobile phase to establish the fingerprint of AP, which has the advantages of environmental friendliness, green, and so on [89]. It makes up for the shortcomings of common organic reagents used in the past to pollute the environment [33]. And, other methods are also often used in the detection of coumarin compounds. Among them, HPLC is the one of the most frequently used analysis methods, and it is also the main method for the analysis of other compounds in AP.

In addition, most of these methods have the advantages of rapidness, precision, accuracy, high sensitivity, short analysis time, strong separation ability, good selectivity, simple operation, low detection line, and wide application range. However, most of the methods have the disadvantages of complex sample pretreatment, high-end instruments and equipment, expensive price, use of toxic organic solvents, pollution of environmental soil, not green and environmentally friendly enough, and so on. But, we believe that with the development of science and technology, more and more low-cost and efficient instruments and green reagents will be developed. 
<smiles>[R]C([R])(C)C1Cc2c(ccc3ccc(=O)oc23)O1</smiles>

$6 \mathrm{R}_{1}=\mathrm{H} ; \mathrm{R}_{2}=\mathrm{CH}_{2} \mathrm{OH}$

$9 \mathrm{R}_{1}=\mathrm{COCH}=\mathrm{CH}\left(\mathrm{C}_{6} \mathrm{H}_{4}\right) \mathrm{OH} ; \mathrm{R}_{2}=\mathrm{CH}_{3}$

$35 \mathrm{R}_{1}=\mathrm{CO}\left(\mathrm{CH}_{3}\right)=\mathrm{CHCH}_{3} ; \mathrm{R}_{2}=\mathrm{CH}_{3}$

$36 \mathrm{R}_{1}=\mathrm{H} ; \mathrm{R}_{2}=\mathrm{CH}_{3}$

$37 \mathrm{R}_{1}=\mathrm{COCH}_{3} ; \mathrm{R}_{2}=\mathrm{CH}_{3}$

$38 \mathrm{R}_{1}=\mathrm{Glu} ; \mathrm{R}_{2}=\mathrm{CH}_{3}$

$39 \mathrm{R}_{1}=\mathrm{COCH}_{2} \mathrm{CH}_{3} ; \mathrm{R}_{2}=\mathrm{CH}_{3}$

$40 \mathrm{R}_{1}=$ Glu-Glu; $\mathrm{R}_{2}=\mathrm{CH}_{3}$

$46 \mathrm{R}_{1}=\mathrm{COCH}\left(\mathrm{CH}_{3}\right) \mathrm{CH}_{2} \mathrm{CH}_{3} ; \mathrm{R}_{2}=\mathrm{CH}_{3}$<smiles>[R20]c1c([R])c([R3])c2oc(=O)ccc2c1[2H]</smiles><smiles>[R3]c1c2ccc(=O)oc2c([R3])c2ccoc12</smiles>

$29 \mathrm{R}_{1}=\mathrm{OCH}_{3} ; \mathrm{R}_{2}=\mathrm{H}$

$30 \mathrm{R}_{1}=\mathrm{OH} ; \mathrm{R}_{2}=\mathrm{H}$

$32 \mathrm{R}_{1}=\mathrm{OCH}_{3} ; \mathrm{R}_{2}=\mathrm{OCH}_{2} \mathrm{CH}(\mathrm{OH}) \mathrm{C}(\mathrm{OH})\left(\mathrm{CH}_{3}\right)_{2}$

$34 \mathrm{R}_{1}=\mathrm{OCH}_{2} \mathrm{CH}=\mathrm{C}\left(\mathrm{CH}_{3}\right) 2 ; \mathrm{R}_{2}=\mathrm{OCH}_{3}$

$47 \mathrm{R}_{1}=\mathrm{OCH}_{3} ; \mathrm{R} 2=\mathrm{OCH}_{2} \mathrm{CHOC}\left(\mathrm{CH}_{3}\right)_{2}$

$50 \mathrm{R}_{1}=\mathrm{H} ; \mathrm{R}_{2}=\mathrm{OCH}_{2} \mathrm{CH}=\mathrm{C}\left(\mathrm{CH}_{3}\right)_{2}$

$52 \mathrm{R}_{1}=\mathrm{OCH}_{2} \mathrm{CH}=\mathrm{C}\left(\mathrm{CH}_{3}\right)_{2} ; \mathrm{R}_{2}=\mathrm{H}$

$55 \mathrm{R}_{1}=\mathrm{OCH}_{2} \mathrm{COCH}\left(\mathrm{CH}_{3}\right)_{2} ; \mathrm{R}_{2}=\mathrm{H}$

$56 \mathrm{R}_{1}=\mathrm{R}_{2}=\mathrm{OCH}_{3}$

$61 \mathrm{R}_{1}=\mathrm{OCH}_{3} ; \mathrm{R}_{2}=\mathrm{OCH}_{2} \mathrm{CH}(\mathrm{OH}) \mathrm{C}\left(\mathrm{CH}_{3}\right)=\mathrm{CH}_{2}$

$66 \mathrm{R}_{1}=\mathrm{OCH}_{2} \mathrm{CHOC}\left(\mathrm{CH}_{3}\right)_{2} ; \mathrm{R}_{2}=\mathrm{H}$

$67 \mathrm{R}_{1}=\mathrm{OCH}_{2} \mathrm{CH}(\mathrm{OH}) \mathrm{C}(\mathrm{OC})\left(\mathrm{CH}_{3}\right)_{2} ; \mathrm{R}_{2}=\mathrm{H}$

$68 \mathrm{R}_{1}=\mathrm{OCH}_{2} \mathrm{CH}(\mathrm{OH}) \mathrm{C}\left(\mathrm{CH}_{3}\right)=\mathrm{CH}_{2} ; \mathrm{R}_{2}=\mathrm{H}$

$70 \mathrm{R}_{1}=\mathrm{R}_{2}=\mathrm{H}$

$72 \mathrm{R}_{1}=\mathrm{OCH}_{3} ; \mathrm{R}_{2}=\mathrm{OCH}_{2} \mathrm{CH}(\mathrm{OAc}) \mathrm{C}(\mathrm{OH})\left(\mathrm{CH}_{3}\right)_{2}$

$73 \mathrm{R}_{1}=\mathrm{OCH}_{3} ; \mathrm{R}_{2}=\mathrm{OCH}(\mathrm{O}-\mathrm{Glu}) \mathrm{CH}_{2} \mathrm{C}\left(\mathrm{CH}_{3}\right)_{2} \mathrm{OH}$

$74 \mathrm{R}_{1}=\mathrm{OCH}_{3} ; \mathrm{R}_{2}=\mathrm{OCH}_{2} \mathrm{CH}(\mathrm{OH}) \mathrm{C}\left(\mathrm{CH}_{3}\right)_{2}$-O-Glu

$79 \mathrm{R}_{1}=\mathrm{H} ; \mathrm{R}_{2}=\mathrm{OCH}_{3}$

$5 \mathrm{R}_{1}=\mathrm{COCH}=\mathrm{C}\left(\mathrm{CH}_{3}\right)_{2} ; \mathrm{R}_{2}=\mathrm{CH}_{3} ; \mathrm{R}_{3}=\mathrm{H} ; \mathrm{R}_{4}=\mathrm{OCH}_{3}$

$7 \mathrm{R}_{1}=\mathrm{COCH}=\mathrm{C}\left(\mathrm{CH}_{3}\right)_{2} ; \mathrm{R}_{2}=\mathrm{CH}_{3} ; \mathrm{R}_{3}=\mathrm{H} ; \mathrm{R}_{4}=\mathrm{OCH}_{2} \mathrm{CH}=\mathrm{C}(\mathrm{CH} 3)_{2}$

$8 \mathrm{R}_{1}=\mathrm{R}_{4}=\mathrm{H} ; \mathrm{R}_{2}=\mathrm{CH}_{3} ; \mathrm{R}_{3}=\mathrm{CH}(\mathrm{OH}) \mathrm{CH}(\mathrm{OH}) \mathrm{C}(\mathrm{OH})\left(\mathrm{CH}_{3}\right)_{2}$

$27 \mathrm{R}_{1}=\mathrm{R}_{3}=\mathrm{R}_{4}=\mathrm{H} ; \mathrm{R}_{2}=\mathrm{O}$-Glu-Api

$41 \mathrm{R}_{1}=\mathrm{CH}_{2} \mathrm{CH}=\mathrm{C}\left(\mathrm{CH}_{3}\right)_{2} ; \mathrm{R}_{2}=\mathrm{CH}_{3} ; \mathrm{R}_{3}=\mathrm{H} ; \mathrm{R}_{4}=\mathrm{OCH}_{3}$

$45 \mathrm{R}_{1}=\mathrm{CH}_{2} \mathrm{CH}_{2} \mathrm{C}(\mathrm{OH})\left(\mathrm{CH}_{3}\right)_{2} ; \mathrm{R}_{2}=\mathrm{CH}_{3} ; \mathrm{R}_{3}=\mathrm{R}_{4}=\mathrm{H}$

$48 \mathrm{R}_{1}=\mathrm{COCH}_{2} \mathrm{C}(\mathrm{OH})\left(\mathrm{CH}_{3}\right)_{2} ; \mathrm{R}_{2}=\mathrm{CH}_{3} ; \mathrm{R}_{3}=\mathrm{H} ; \mathrm{R}_{4}=\mathrm{OCH}_{3}$

$59 \mathrm{R}_{1}=\mathrm{CH}_{2} \mathrm{CH}(\mathrm{OH}) \mathrm{C}(\mathrm{OH})\left(\mathrm{CH}_{3}\right)_{2} ; \mathrm{R}_{2}=\mathrm{CH}_{3} ; \mathrm{R}_{3}=\mathrm{R}_{4}=\mathrm{H}$

$60 \mathrm{R}_{1}=\mathrm{COCH}=\mathrm{C}\left(\mathrm{CH}_{3}\right)_{2} ; \mathrm{R}_{2}=\mathrm{CH}_{3} ; \mathrm{R}_{3}=\mathrm{R}_{4}=\mathrm{H}$

$64 \mathrm{R}_{1}=\mathrm{CH}_{2} \mathrm{CH}=\mathrm{C}\left(\mathrm{CH}_{3}\right)_{2} ; \mathrm{R}_{2}=\mathrm{CH}_{3} ; \mathrm{R}_{3}=\mathrm{R}_{4}=\mathrm{H}$

$65 \mathrm{R}_{1}=\mathrm{CHCH}=\mathrm{C}\left(\mathrm{CH}_{3}\right)_{2} ; \mathrm{R}_{2}=\mathrm{CH}_{3} ; \mathrm{R}_{3}=\mathrm{R}_{4}=\mathrm{H}$

$69 \mathrm{R}_{1}=\mathrm{R}_{2}=\mathrm{R}_{4}=\mathrm{H} ; \mathrm{R}_{3}=\mathrm{CH}_{2} \mathrm{CH}(\mathrm{OH}) \mathrm{C}(\mathrm{OH})\left(\mathrm{CH}_{3}\right)_{2}$

$71 \mathrm{R}_{1}=\mathrm{R}_{2}=\mathrm{R}_{4}=\mathrm{H} ; \mathrm{R}_{3}=\mathrm{OCH}_{3}$

$75 \mathrm{R}_{1}=\mathrm{R}_{4}=\mathrm{H} ; \mathrm{R}_{2}=\mathrm{CH}_{3} ; \mathrm{R}_{3}=\mathrm{CHCH}(\mathrm{OH}) \mathrm{C}(\mathrm{OH})\left(\mathrm{CH}_{3}\right)_{2}$

$76 \mathrm{R}_{1}=\mathrm{R}_{2}=\mathrm{R}_{3}=\mathrm{R}_{4}=\mathrm{H}$

$77 \mathrm{R}_{1}=\mathrm{R}_{2}=\mathrm{R}_{4}=\mathrm{H} ; \mathrm{R}_{2}=\mathrm{CH}_{2} \mathrm{CH}=\mathrm{C}\left(\mathrm{CH}_{3}\right) \mathrm{CH}_{2} \mathrm{CH}_{2} \mathrm{CH}=\mathrm{C}\left(\mathrm{CH}_{3}\right) \mathrm{CH}_{2} \mathrm{CH}_{2} \mathrm{C}=\mathrm{C}\left(\mathrm{CH}_{3}\right)_{2}$<smiles>CC=C(C)C(=O)OC(c1cc2ccc(=O)oc2cc1OC)C1OC(C)(C)OC1(C)C</smiles>

23

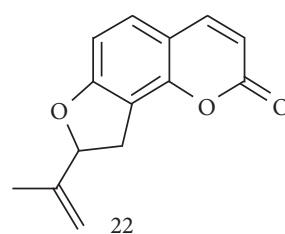

22

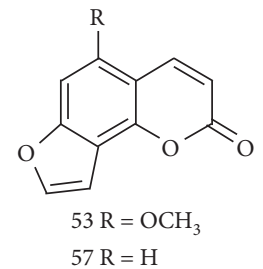

$\mathrm{HO}$<smiles>[R20]O[C@H](c1cc2ccc(=O)oc2cc1OC)[C@@H]([R20])C(C)(C)C</smiles>

$10 \mathrm{R}_{1}=\mathrm{H}, \mathrm{R}_{2}=$ tigloyl

$16 \mathrm{R}_{1}=$ angeloyl; $\mathrm{R}_{2}=\mathrm{H}$

$19 \mathrm{R}_{1}=\mathrm{CH}_{2} \mathrm{CH}_{3} ; \mathrm{R}_{2}=\mathrm{H}$<smiles>[R20]O[C@H](c1cc2ccc(=O)oc2cc1OC)[C@@H](O[R20])C(C)(C)O</smiles>

$11 \mathrm{R}_{1}=\mathrm{H}, \mathrm{R}_{2}=$ tigloyl $12 \mathrm{R}_{1}=\mathrm{H} ; \mathrm{R}_{2}=$ methybutyryl $13 \mathrm{R}_{1}=$ tigloyl; $\mathrm{R}_{2}=\mathrm{H}$ $18 \mathrm{R}_{1}=\mathrm{H} ; \mathrm{R}_{2}=$ isovalery $20 \mathrm{R}_{1}=$ angeloyl; $\mathrm{R}_{2}=\mathrm{H}$ $21 \mathrm{R}_{1}=$ isovalery; $\mathrm{R}_{2}=\mathrm{H}$<smiles>[R]C(c1cc2ccc(=O)oc2cc1OC)C(O)C(C)(C)O</smiles>

$25 \mathrm{R}=\mathrm{OCOCH}_{2} \mathrm{CH}\left(\mathrm{CH}_{3}\right)_{2}$ $54 \mathrm{R}=\mathrm{OCOC}\left(\mathrm{CH}_{3}\right)=\mathrm{CHCH}_{3}$<smiles>[R]C(=C([R])C(C)(C)O)c1cc2ccc(=O)oc2cc1OC</smiles>

$42 \mathrm{R}_{1}=\mathrm{H} ; \mathrm{R}_{2}=\mathrm{OCOC}\left(\mathrm{CH}_{3}\right)=\mathrm{CHCH}_{3}$ $44 \mathrm{R}_{1}=\mathrm{H} ; \mathrm{R}_{2}=\operatorname{OCOCH}\left(\mathrm{CH}_{3}\right) \mathrm{CH}_{2} \mathrm{CH}_{3}$

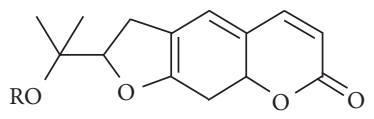

$58 \mathrm{R}=\mathrm{Glu}$ $62 \mathrm{R}=\mathrm{H}$

FIgURE 1: The structures of coumarin compounds of AP.

The species of AP are complex and difficult to distinguish, which are easily affected by the growing environment, harvesting time, processing methods, and storage conditions. Moreover, the content of active components in AP is different in different areas. Due to these factors, the quality and clinical efficacy of AP cannot be controlled at present.

Currently, the 2015 edition of Chinese Pharmacopoeia uses TLC for the qualitative identification of AP and HPLC for the determination of the content of osthol and columbianadin. Moreover, it is stipulated that AP should contain osthol no less than $0.50 \%$ and contain columbianadin no less than $0.080 \%$.

However, TCM has the characteristics of multicomponent and multitarget. It is limited to evaluate the quality of TCM only by the content of one or two compounds. In order to evaluate the quality of AP, Yang et al. used the UPLCPDA method to determine the content of 6 phenolic acids and 7 coumarin compounds [90]. Ding et al. determined the 
TABLE 3: The volatile oils compounds of AP.

\begin{tabular}{|c|c|c|}
\hline No. & Compounds & Reference \\
\hline 1 & Acorenone & {$[61]$} \\
\hline 2 & Aristolene & [49] \\
\hline 3 & Benzene-1-methyl-2-(1-methylethyl) & [49] \\
\hline 4 & Benzene-2-methoxy-4-methyl-1-(1-methylethyl) & {$[49]$} \\
\hline 5 & Benzene-1,2,4-trimethoxy-5-(1-proprnyl)) & [49] \\
\hline 6 & $\alpha$-Bergamotene & {$[43]$} \\
\hline 7 & Bicyclo- $[3,10]$-hexan-3-ol & [49] \\
\hline 8 & Bicycol $[7,2,0]$ undec-4-ene, 4,11,11-trimethyl-8-methylene & {$[43]$} \\
\hline 9 & $\alpha$-Bisabolene & {$[43]$} \\
\hline 10 & $\beta$-Bisabolene & {$[43]$} \\
\hline 11 & Camphene & {$[44]$} \\
\hline 12 & 6-Camphenone & {$[49]$} \\
\hline 13 & 3-Carene & {$[48]$} \\
\hline 14 & Cedrol & [49] \\
\hline 15 & $\alpha$-Copaene & {$[43]$} \\
\hline 16 & p-Cresol & {$[43]$} \\
\hline 17 & Cyclohexene & [49] \\
\hline 18 & $\alpha$-Cyclohexyl decane & {$[43]$} \\
\hline 19 & 1,3-Cyclohexadiene-5-(1,5-dimethyl-4-hexenyl-2-methyl & {$[43]$} \\
\hline 20 & $(+)$-Cycloisosativene & [49] \\
\hline 21 & 2-Cyclopropen-1-one,2,3-diphenyl & {$[43]$} \\
\hline 22 & Decylacetate & {$[44]$} \\
\hline 23 & [s-(R, s)]-3-(1,5 Dimethyl-4-hexene)-6-methylene-cyclohexene & {$[44]$} \\
\hline 24 & 3,6-Dimethyloctane & [49] \\
\hline 25 & 2,2-Dimethyl-8-oxo-3,4-dihydro-2H,8H-pyrano[3,2-g] chromen-3-yl ester & {$[50]$} \\
\hline 26 & 8,8-Dimethyl-2-oxo-2,8,9,10-tetrahydropyrano(2,3-f)chromene- 9,10 -diylbis & {$[50]$} \\
\hline 27 & 5,6-Dimethyl-3a,4,7,7a-H & {$[43]$} \\
\hline 28 & $\alpha$-Elemene & {$[43]$} \\
\hline 29 & $\beta$-Elemene & {$[43]$} \\
\hline 30 & Elemol & {$[50]$} \\
\hline 31 & Elixene & {$[43]$} \\
\hline 32 & Eremophilene & {$[43]$} \\
\hline 33 & [1S-(1 $\alpha, 2 \beta, 4 \beta)]$-1-Ethenyl-1-methyl-2,4-bis-(1-methyethenyl)-cyclohexane & {$[44]$} \\
\hline 34 & $\alpha$-Funebrene & {$[43]$} \\
\hline 35 & Globulol & [49] \\
\hline 36 & Guaiol & {$[43]$} \\
\hline 37 & $\beta$-Gurjunene & {$[50]$} \\
\hline 38 & (Z)-7-Hexadecenal & [49] \\
\hline 39 & 1-Hexadecanol & {$[50]$} \\
\hline 40 & 3-Hexanol & {$[44]$} \\
\hline 41 & Humulene & {$[43]$} \\
\hline 42 & 4-Hydroxy-3-methylacetophenone & [47] \\
\hline 43 & 1,3-Isobenzafurandione,3a,4,7,7a-tetrahydro-5,6-dimethyl & {$[51]$} \\
\hline 44 & Isopropy-4-methylene-7-methyl-1, 2, 3,4, 4A, 5. 6, 8A-octahydronaphthalene & {$[43]$} \\
\hline 45 & Isosericenine & {$[43]$} \\
\hline 46 & Ledol & [49] \\
\hline 47 & Leecanoic acid,2,4,6-trirnethyl-methyl ester & [43] \\
\hline 48 & Lignoceric acid & {$[51]$} \\
\hline 49 & $d$-Limonene & {$[48]$} \\
\hline 50 & 4-Longifolene & [49] \\
\hline 51 & $\alpha$-Longipinene & {$[43]$} \\
\hline 52 & 4-Methoxy-6-(2-propenyl)-1,3-benzodioxole & [49] \\
\hline 53 & 4-Methoxyacetophenone & {$[43]$} \\
\hline 54 & 8-Methyl-1-decene & {$[44]$} \\
\hline 55 & 3-Methyl-but-2-enoic acid & {$[50]$} \\
\hline 56 & 4-Methylclohexanone & {$[43]$} \\
\hline 57 & 1-Methyl-1,4-cyclohexadiene & {$[44]$} \\
\hline 58 & 4-Methylene-1-(1-methylethyl)-bicyclo $[3,1,0]$ hexane & {$[44]$} \\
\hline 59 & 4-Methylene-1-(1-methylethyl)-bicyclo $[3,1,0]$ hex-2-ene & {$[44]$} \\
\hline 60 & 4-Methylene-1,5,5-trimethylcyclohecene & {$[44]$} \\
\hline
\end{tabular}


TABLE 3: Continued.

\begin{tabular}{|c|c|c|}
\hline No. & Compounds & Reference \\
\hline 61 & 4,4'-Methylenebis[2,3,5,6-tetremethyl] phenol & {$[43]$} \\
\hline 62 & 2-[1-Methylethyl-methylcarbam ate] phenol & [49] \\
\hline 63 & 2-Methyl-1-methylene-3-(1-methylethenyl)-cyclopentane & {$[44]$} \\
\hline 64 & 2-Methyl-5-(1-methylethyl)-phenol & [49] \\
\hline 65 & 3-Methylnolane & {$[43]$} \\
\hline 66 & 2-Methyloctane & {$[43]$} \\
\hline 67 & o-Methylphenol & {$[49]$} \\
\hline 68 & 9-Methyl-1-undecene & {$[49]$} \\
\hline 69 & 10-Methyl-1-undecene & {$[49]$} \\
\hline 70 & Myrcene & [43] \\
\hline 71 & Myrtenal & {$[43]$} \\
\hline 72 & 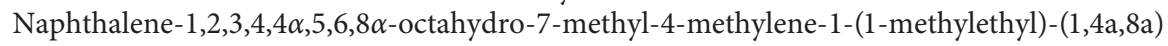 & {$[43]$} \\
\hline 73 & Naphthalenone & [49] \\
\hline 74 & Nerolidol & {$[43]$} \\
\hline 75 & n-Nonane & {$[43]$} \\
\hline 76 & n-Octane & {$[43]$} \\
\hline 77 & n-Undecane & {$[43]$} \\
\hline 78 & 9,12-Octadecadienoic acid methyl ester & [49] \\
\hline 79 & o-Cymene & [47] \\
\hline 80 & Osthol & {$[43]$} \\
\hline 81 & Oxacyclohexadecan- $\alpha$-one & {$[43]$} \\
\hline 82 & $\gamma$-Patchoulene & {$[43]$} \\
\hline 83 & p-Cresol & {$[51]$} \\
\hline 84 & p-Cymene & {$[9]$} \\
\hline 85 & 7,10 -Pentadecadiynoic acid & {$[43]$} \\
\hline 86 & 1-Penten-3-one thyl vinyl ketone & {$[43]$} \\
\hline 87 & Pentylbenzene & [49] \\
\hline 88 & Peucedanol & {$[43]$} \\
\hline 89 & Phenol,4-(1,1-dimethylethyl)-2-methyl & {$[43]$} \\
\hline 90 & $\alpha$-Phellandrene & {$[43]$} \\
\hline 91 & $\alpha$-Pinene & {$[43]$} \\
\hline 92 & $\beta$-Pinene & {$[43]$} \\
\hline 93 & $\alpha$-Selinene & {$[47]$} \\
\hline 94 & $\beta$-Sesquiphellandrene & [47] \\
\hline 95 & Sylvestrene & {$[43]$} \\
\hline 96 & $\alpha$-Terpinene & {$[43]$} \\
\hline 97 & 1-Tetradecene & {$[43]$} \\
\hline 98 & 1,5,5,8-Tetramethyl-12-oxabicyclo $[9,1,0]$ dodeca-3,7-diene & {$[44]$} \\
\hline 99 & Thalic acid diisobuthyl ester & {$[49]$} \\
\hline 100 & Thymol & [43] \\
\hline 101 & Toluene & {$[49]$} \\
\hline 102 & trans-p-Menth-2-en-7-ol & {$[51]$} \\
\hline 103 & 3, 7, 11-Tridecatrienoic acid,4,8,12-trimethl-ester[Z,E] & {$[43]$} \\
\hline 104 & 1,7,7-Trimethylbicyclo-[2.2.1]-hept-2-yl-acetate & {$[50]$} \\
\hline 105 & 2,4,6-Trimethylmethyl ester-decanoic acid & {$[43]$} \\
\hline 106 & 1,3,3-Trimethyl-tricyclo[2.2.1.02,6] heptane & {$[44]$} \\
\hline 107 & $7 \alpha$-Twtrahydro-5,6-dimethyl & {$[43]$} \\
\hline 108 & Ulopterol & {$[43]$} \\
\hline 109 & Zingiberene & [49] \\
\hline
\end{tabular}

content of coumarin by LC-MS/MS [91]. Yang et al. used the UPLC-MS/MS method to determine the content of 15 compounds in AP and found that columbianetin acetate, osthol, chlorogenic acid, and psoralen were the main components causing the differences in AP from different sources. Hou et al. established the UPLC fingerprint of AP, which again proved that columbianetin acetate and osthol were the main components that caused the difference of AP in different areas [92]. However, there are few methods to evaluate the quality of AP in relation to its pharmacological activity. It is obviously not enough to evaluate AP quality solely on the basis of chemical components. More methods should be established by quality evaluation related to activity or based on chemical activity.

\section{Processing Method}

Processing can remove nonmedicinal parts, keep the active ingredients, make the drug pure, reduce toxicity, detoxify, and change the drug ingredients to enhance the efficacy. 
TABLE 4: The organic acid compounds of AP.

\begin{tabular}{lcc}
\hline No. & Compounds & Reference \\
\hline 1 & Caffeic acid & {$[43]$} \\
2 & 3-O-Caffeoylquinic acid & {$[71]$} \\
3 & 4-O-Caffeoylquinic acid & {$[71]$} \\
4 & 5-O-Caffeoylquinic acid & {$[71]$} \\
5 & Ferulic acid & {$[71]$} \\
6 & $1,5-$ Dicaffeoylquinic acid & {$[71]$} \\
7 & $3,4-$ Dicaffeoylquinic acid & {$[71]$} \\
8 & 3,5-Dicaffeoylquinic acid & {$[71]$} \\
9 & $4,5-$ Dicaffeoylquinic acid & {$[71]$} \\
10 & Isoferulic acid & {$[44]$} \\
11 & {$[2,3,4,9-$ Tetrahydro-1H-pyrido-(3,4-b)]-indole-3-(ar-boxy-lic acid) } & {$[71]$} \\
12 & 3-O-trans-Coumaroylquinic acid & {$[44]$} \\
13 & 3-O-trans-Feruloylquinic acid & {$[44]$} \\
\hline
\end{tabular}<smiles>[R]c1cc(Br)c(O)cc1Br</smiles>

$1 \mathrm{R}_{1}=\mathrm{H} ; \mathrm{R}_{2}=\mathrm{OH} ; \mathrm{R}_{3}=\mathrm{CH}=\mathrm{CHCOOH}$ $5 \mathrm{R}_{1}=\mathrm{CH}=\mathrm{CHCOOH} ; \mathrm{R}_{2}=\mathrm{OCH}_{3} ; \mathrm{R}_{3}=\mathrm{H}$ $10 \mathrm{R}_{1}=\mathrm{H} ; \mathrm{R}_{2}=\mathrm{OCH}_{3} ; \mathrm{R}_{3}=\mathrm{CH}=\mathrm{CHCOOH}$ $12 \mathrm{R}_{1}=\mathrm{CH}=$ CHCOO-quinic; $\mathrm{R}_{2}=\mathrm{R}_{3}=\mathrm{H}$ $13 \mathrm{R}_{1}=\mathrm{CH}=\mathrm{CHCOO}$-quinic; $\mathrm{R}_{2}=\mathrm{OCH}_{3} ; \mathrm{R}_{3}=\mathrm{H}$<smiles>O=C(/C=C/c1ccc(O)c(O)c1)O[C@@H]1C[C@@](O)(C(=O)O)C[C@H](O)[C@H]1O</smiles><smiles>O=C(/C=C/c1ccc(O)c(O)c1)O[C@H]1[C@@H](O)C[C@@](O)(C(=O)O)C[C@H]1O</smiles><smiles>O=C(/C=C/c1ccc(O)c(O)c1)O[C@H]1C[C@@](O)(C(=O)O)C[C@H](O)[C@H]1O</smiles><smiles>O=C(/C=C/c1ccc(O)c(O)c1)O[C@H]1C[C@@](OC(=O)/C=C/c2ccc(O)c(O)c2)(C(=O)O)C[C@H](O)[C@H]1O</smiles><smiles>O=C(/C=C/c1ccc(O)c(O)c1)O[C@H]1[C@@H](O)C[C@@](O)(C(=O)O)C[C@H]1OC(=O)/C=C/c1ccc(O)c(O)c1</smiles><smiles>O=C(/C=C/c1ccc(O)c(O)c1)O[C@H]1C[C@](O)(C(=O)O)C[C@H](OC(=O)/C=C/c2ccc(O)c(O)c2)[C@@H]1O</smiles><smiles>O=C(/C=C/c1ccc(O)c(O)c1)O[C@H]1CC[C@@](O)(C(=O)O)C[C@H]1OC(=O)/C=C/c1ccc(O)c(O)c1</smiles>

9<smiles>O=C(O)[C@@H]1Cc2c([nH]c3ccccc23)CN1</smiles>

11

FIgURE 2: The structures of organic acid compounds of AP.

Thunder gong processing theory (雷公炮多论) recorded that there was a steaming method of epimedium in the Northern and Southern dynasties. Lishang Xuduan Fang (理伤续断 方) recorded that there was the method of removing reed in the Tang dynasty. The Song dynasty had to collect the reed and then wash and bake them. Decoction and Material Medica (汤液本草) recorded there was a method of peeling and then washing in the Yuan dynasty. In the Ming dynasty, salt water immersion baking was practiced (Prescriptions for Universal Relief (普济方)), and also removing section to fry (Surgical Cases (外科理例)) and wine wash method
(Curative Measures for All Diseases (万病回春)). The Qing dynasty has the wine stir-fry (Chuanyabu (串雅补)), the wine immersion method (Gynecology jade ruler (妇科玉 尺)), and so on. Currently, there are net preparation, cut preparation (2015 edition Chinese Pharmacopoeia), and frying method (Integration of Medicine (医学集成)).

\section{Pharmacological Effects}

As a traditional Chinese medicine, AP has a variety of effects, such as anti-inflammatory [93], anti-rheumatism, sedation 
[94], hypnosis, neuroprotection [95], antioxidation [96], antitumor [97], and antigastric ulcer [98], which has been studied and confirmed by many scholars. At present, the pharmacological effects of AP are summarized in Table 5, which is described in detail as follows.

7.1. Analgesic, Sedative, and Anti-Inflammatory Effect. The decoction of AP has sedative and hypnotic effects on rats and mice and can prevent the convulsive effect of strychnine on frogs but it cannot prevent them from dying. Li et al. studied the anti-inflammatory effect of the AP ethanol extract of different concentrations on cotton ball implantation and found that $60 \%$ and $80 \%$ ethanol extract had anti-inflammatory effect $(P<0.05)$, while $40 \%$ ethanol extract had no such effect [93]. The fingerprint-effect relationship of AP was established by Hou et al., whose research results showed that the extract of AP had significant inhibitory effects on the pain and inflammatory reactions of mice caused by hot plate $(P<0.01)$, acetic acid writhing $(P<0.01$, the analgesia rate could reach $89.27 \%)$, formalin $(P<0.01$, the analgesia rate could reach 52.91\%), ear swelling $(P<0.01$, the inhibition rate of swelling was up to $69.33 \%$ ), caused by xylene, and foot swelling $(P<0.01$, the inhibition rate of inflammation was $23.49 \%$ ), caused by carrageenan. Even the analgesic and antiinflammatory activity of AP from some places was stronger than that of the positive drug aspirin. The analysis of fingerprint-effect relationship has shown that osthol, bergapten, columbianetin acetate, and isoimperatorin were the main active components with anti-inflammatory and analgesic effect of AP [92]. However, this study used only a single dose and lacked a dose-dependent study.

Fan et al. studied the analgesic and anti-inflammatory effects of the volatile oil components of AP. The anti-inflammatory effects were observed by the swelling of rats' feet caused by egg white, and the analgesic effects were observed by the hot plate method and acetic acid writhing method in mice. The result showed that the components of volatile oil had no obvious analgesic effect on the pain of mice caused by hot plate, the high dose of the AP volatile oil group could significantly reduce the number of writhing of mice caused by acetic acid $(P<0.05$, the analgesia rate could reach $76.8 \%$ ), and the low and high dose of AP volatile oil groups both had a good anti-inflammatory effect on the foot swelling of rats caused by egg white $(P<0.01)$. However, this study only speculated that the anti-inflammatory and analgesic effects of volatile oil in AP were related to the release of 5-HT, and the relevant mechanism was not studied [94].

The study by Qiu et al. found that the ethanol extract of AP had different degrees of inhibition on cyclooxygenase-1 (COX-1) and cyclooxygenase-2 (COX-2). With the increase in the dosage of AP, the inhibitory effect was enhanced, which may also be one of the anti-inflammatory mechanisms of AP [99]. And other studies had shown that AP has analgesic effects by acting on the central and peripheral nervous systems [117]. And, the coumarin compounds in AP could inhibit the increase in peritoneal and skin vascular permeability induced by histamine and bradykinin, suggesting that the anti-inflammatory effect of AP might be related to the inhibition of histamine and bradykinin. Ma established a neural injury model to study the analgesic effect of coumarins in AP, finding that AP has the analgesic effect mainly related to reducing the concentration of proinflammatory cytokines of TNF- $\alpha$, IL- $1 \beta$, and IL- 6 in the neural injury model and reduces the expression of TRPV1 and perk in the damaged neurons, which also indicated that it may be related to the presence of osthol and columbianadin in AP [102].

Sun et al. studied the hydrolytic activity of N-acylethanolamine-hydrolyzing acid amidase (NAAA) and its anti-inflammatory effect on the LPS-induced mouse macrophage RAW 264.7 inflammatory response model. The results showed that the volatile oil of AP could inhibit the hydrolysis activity of NAAA and increase the level of $\mathrm{N}$-palmitoylethanolamine (PEA) in RAW 264.7 cells induced by LPS, thereby downregulating the expression of TNF- $\alpha$, iNOS, and IL- 6 mRNA and inhibiting the release of TNF- $\alpha$ and NO in RAW 264.7 cells to play an anti-inflammatory effect [100]. Li et al. found that angesesquid A and angesesquid $\mathrm{B}$ could inhibit the release of nitric oxide (NO) in the inflammatory model cells of chondrocytes of intervertebral disc in vitro $(P<0.001)$, thus inhibiting the occurrence of inflammatory response. However, both methods lacked a positive control group [101].

7.2. Cardiovascular Effect. The alcohol extract of AP could inhibit the platelet aggregation induced by ADP in rats and the thrombus formed by the Chandler method and could also shorten the length of the thrombus and prolong the time of tail hemorrhage in mice. Moreover, the inhibition rate of platelet aggregation was enhanced with the increase in the AP alcohol extract concentration. When the AP alcohol extract concentration reached $0.4 \mathrm{~g} / \mathrm{kg}$, it had an inhibitory effect on the thrombosis formed by carotid vein bypass in rats $(P<0.05)$, and when the AP alcohol extract concentration reached $1.0 \mathrm{~g} / \mathrm{kg}$, the inhibition rate could reach 51.1\% $(P<0.01)$. However, the method lacked a positive control group and failed to provide reliable data for clinical application. At the same time, only the AP alcohol extract was studied in this study, and there was no research on related bioactive monomer compounds [103].

Some studies had proved that the active ingredients of antiplatelet aggregation and antithrombotic activity were columbianetin acetate, columbianetin, osthol, and columbianedin [118]. Li et al. research showed that gammaaminobutyric acid (GABA) was one of the main active components of AP, which had antiarrhythmic effect. Gamma-aminobutyric acid (10 mg/kg, iv) could prolong the start time of ventricular arrhythmia induced by aconitine in mice (control group $1.7 \pm 0.12 \mathrm{~min}$ and GABA group $2.0 \pm 0.4 \mathrm{~min}, P<0.05)$ and increased the threshold dose (control group $80.9 \pm 9.4 \mathrm{~g} / \mathrm{kg}$ and GABA group $94.3 \pm 15.0 \mathrm{~g} / \mathrm{kg}, \quad P<0.05) . \quad$ Gamma-aminobutyric acid $(10 \mathrm{mg} / \mathrm{kg}$, iv) could delay the start time of arrhythmias induced by aconitine in rats (control group $3.5 \pm 1.1 \mathrm{~min}$, GABA group $13.2 \pm 8.8 \mathrm{~min}, P<0.05)$, reduce the incidence of ventricular tachycardia (control group 100\%, GABA 


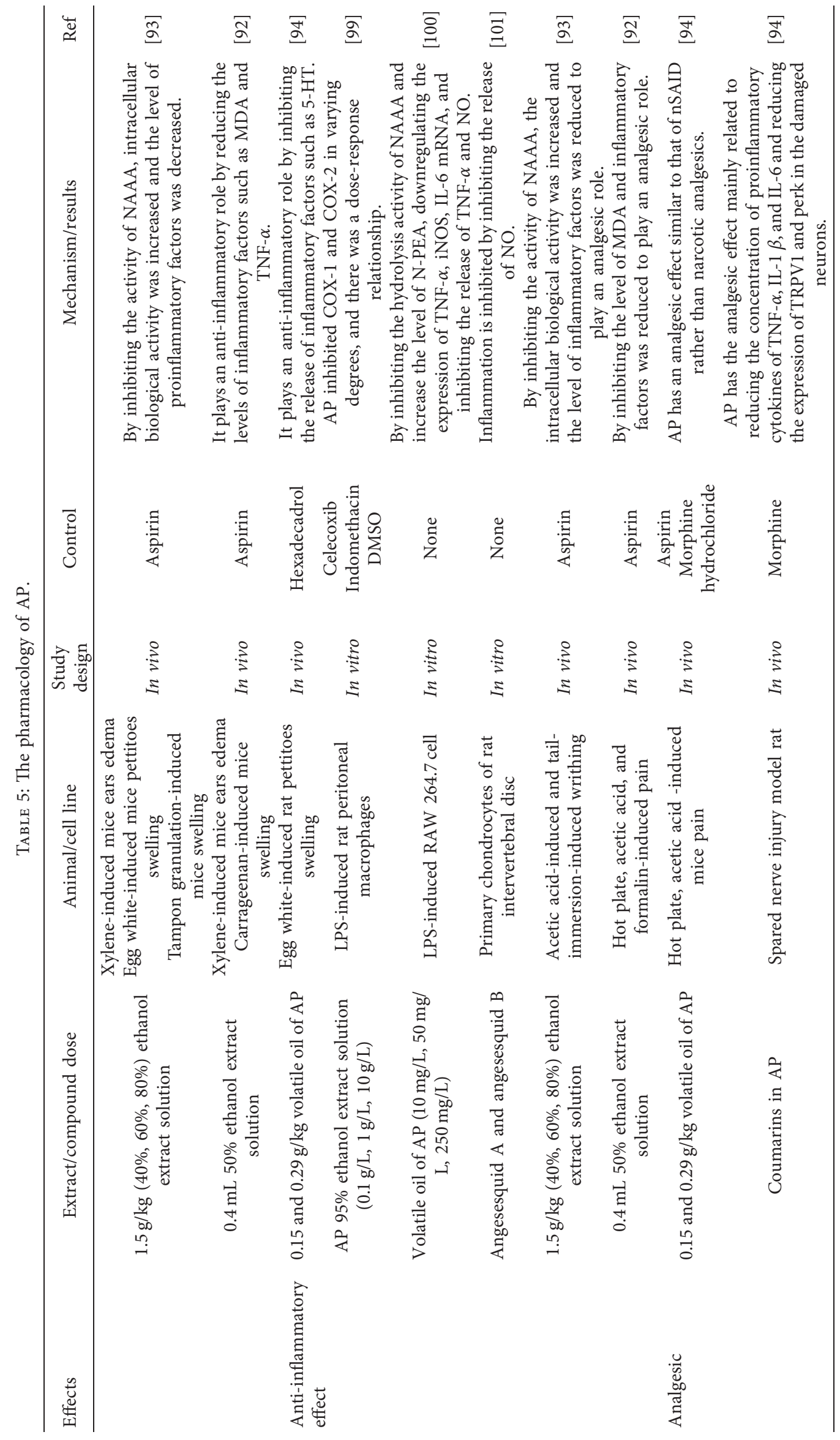




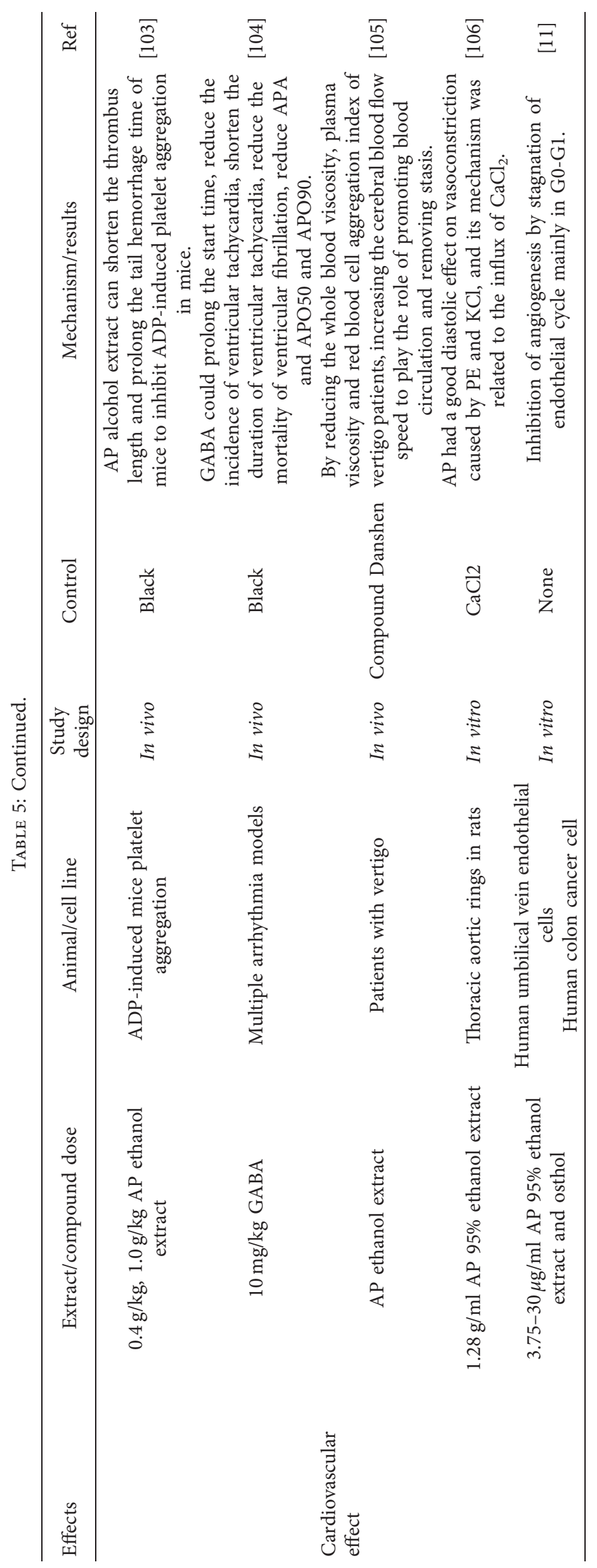




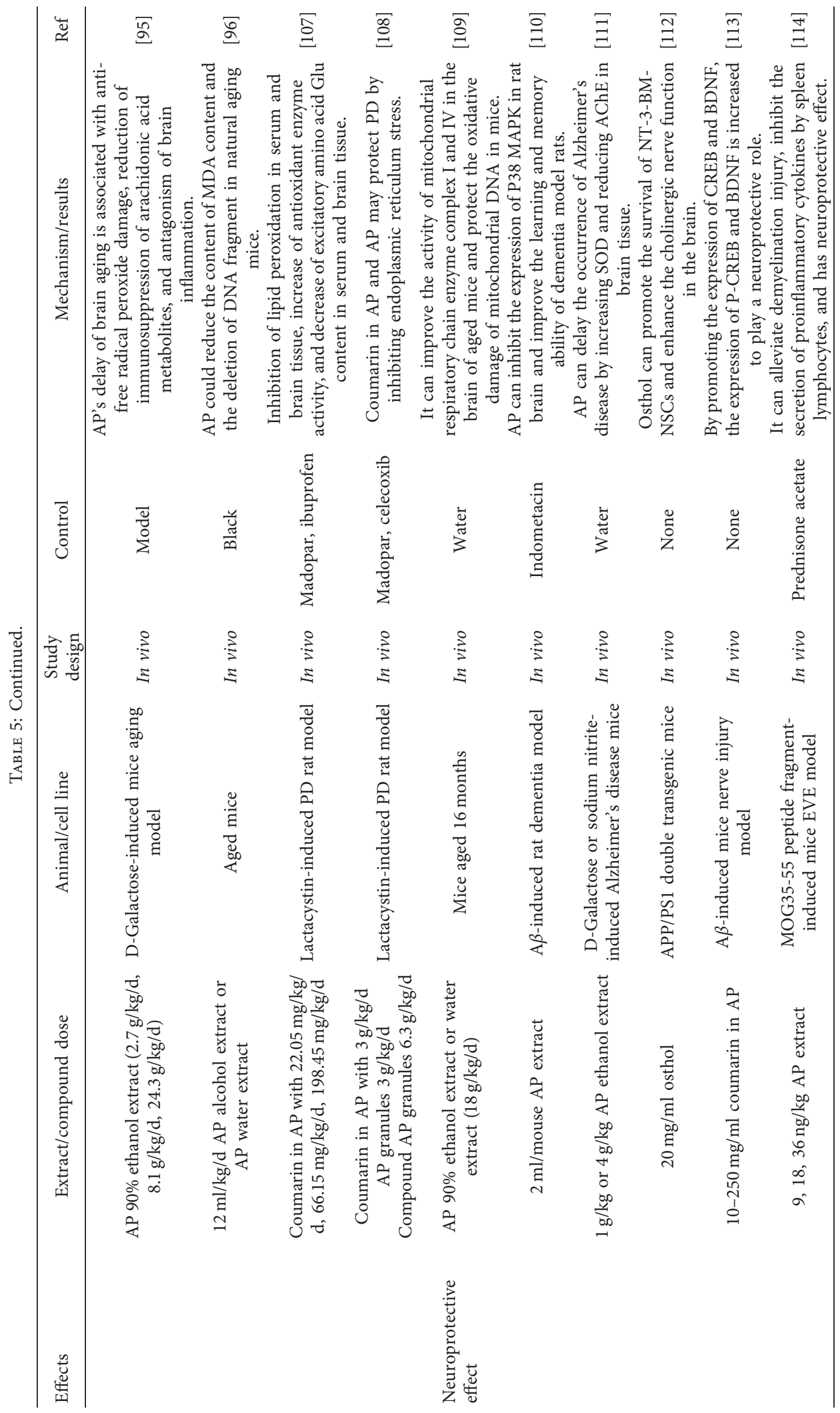




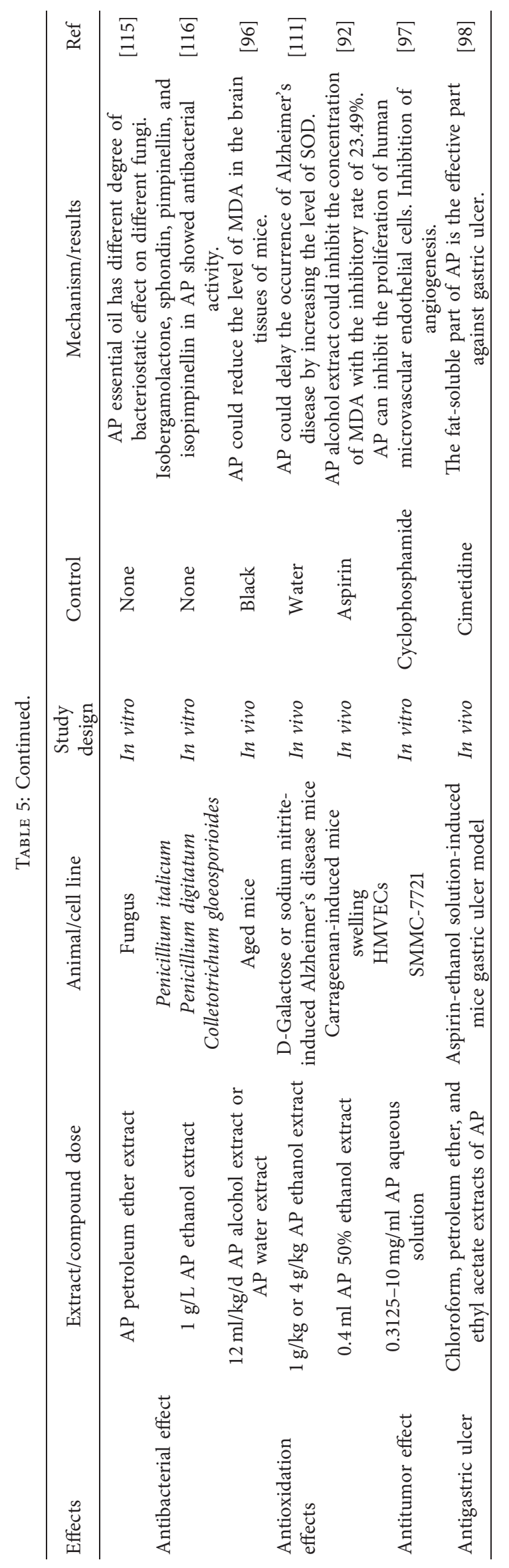


group 50\%, $P<0.05)$, shorten the duration of ventricular tachycardia (control group $22.2 \pm 6.9 \mathrm{~min}$, GABA group $12.3 \pm 0.5 \mathrm{~min}, P<0.05)$, and reduce the mortality of ventricular fibrillation (control group 50\%, $P<0.05$ ). Gammaaminobutyric acid (10 mg/kg, iv) also had an effect on ventricular action potential in rats, which could reduce APA and APO 50 and APO 90. However, this experiment lacked a positive control group and a comparison between the pharmacodynamics of gamma-aminobutyric acid and the AP crude extract [104].

Chen et al. used Compound Danshen as the positive control group to study the function of activate blood and resolve stasis of the AP alcohol extract. It was found that it can also significantly reduce the whole blood viscosity, plasma viscosity, and red blood cell aggregation index of vertigo patients and increase the velocity of blood flow in cerebral vessels. The results showed that AP alcohol extract treatment was effective, with an effective rate of $95.5 \%$, which was significantly higher than the control group $(P<0.05$, the effective rate was $79.5 \%)$. This showed that the AP alcohol extract could play a good role in activating blood and resolving stasis. However, this study only used a single dose for clinical research, lacking dose-dependent studies and studies on the maximum safe dose of AP [105]. We speculate that the possible mechanism of AP is related to dilation of blood vessels, reduction of blood viscosity, and improvement of microcirculation. It may also be related to GABA contained in AP, increase in cardiac pump function, and influence of the cardiac output. The future scholars should also discuss the specific pharmacodynamic substances that can activate blood circulation and remove blood stasis in the ethanol extract and analyze and verify the pharmacodynamic substances and possible metabolites through in vivo and in vitro pharmacological experiments, which will be of certain reference value for future studies.

Some studies had also shown that the AP root water extract at low concentration could effectively inhibit the proliferation of human microvascular endothelial cells [97] Chen and Lu showed that AP had a good diastolic effect on vasoconstriction caused by $\mathrm{PE}$ and $\mathrm{KCl}$, and its mechanism was related to the influx of $\mathrm{CaCl} 2$ [106]. $\mathrm{Hu}$ et al. discussed the inhibitory effect and mechanism of AP on angiogenesis and considered osthol as the main component of antiangiogenesis in vitro. The inhibition rates of treating with $3.75-30 \mu \mathrm{g} / \mathrm{ml}$ AP extract and osthol for $48 \mathrm{~h}$ on cell proliferation of HUVEC were $5.16 \%-10.15 \%$ and $22.64 \%-65.56 \%$ and those of LoVo cells were $2.86 \%-7.29 \%$ and $5.15 \%-24.39 \%$, respectively. The inhibition rates of treating with $3.75-30 \mu \mathrm{g} / \mathrm{ml} \mathrm{AP}$ extract and osthol for $24 \mathrm{~h}$ on HUVEC cell migration were between $2.16 \%-8.00 \%$ and $13.70 \%-63.04 \%$, and the apoptosis rates of HUVEC cells were between $6.1 \%-14.4 \%$ and $18.8 \%-89.5 \%$, respectively. The results showed that the effect of osthol on the cell cycle was stronger than that of the AP alcohol extract [119], which laid a foundation for AP to become a clinical Chinese medicine for antiangiogenesis.

7.3. Neuroprotective Effect. The ethanol extract of AP could improve the learning and memory ability of aging mice by improving the aging of phospholipid components in different parts of the cerebral cortex, hippocampus, and striatum, increasing the concentration of phosphatidylcholine (PC), reducing the content of sphingomyelins (SM), and improving the aging changes of the thymus and hippocampus [94]. It also shown that the mechanism of AP delaying brain aging was related to its antiperoxidation damage of free radicals, reducing the immunosuppression of arachidonic acid metabolites and antagonizing brain inflammation [94]. A study showed that brain aging was related to the increase in malondialdehyde (MDA) content and DNA fragment deletion in brain tissues, and the AP decoction and alcohol extract could reduce the content of MDA and the deletion of the DNA fragment in natural aging mice [96]. Moreover, the effect of the alcohol extract was better than that of the water extract, which may be related to the more effective component coumarin in the alcohol extract [120]. Pei et al. found that the mechanism of the neuroprotective effect of coumarin in AP was related to the inhibition of the content of excitatory amino acid Glu in brain tissues and serum and the inhibition of the expression of the stress factors $\mathrm{CHOP}$ and Caspase12 in the substantia nigra in brain tissues $[107,108,121]$. Li et al. found that AP could significantly improve the activity of mitochondrial respiratory chain enzyme complex I and IV in the brain of aged mice and had a protective effect on the oxidative damage of mitochondrial DNA in mice [109]. Zhang et al. showed that AP could inhibit the expression of IL-1, IL-6, TNF- $\alpha$, p38MAPK, and iNOS in rat brain tissue by regulating $\mathrm{NF}-\kappa \mathrm{B}$ and increase the ratio of $\mathrm{Bcl}-2 / \mathrm{Bax}$ to reduce the occurrence of inflammation, apoptosis, oxidative stress, and other reactions in brain tissue. AP could also play a neuroprotective role by inhibiting the ability of spleen lymphocytes to secrete proinflammatory factors and increasing the expression of the neurofilament protein [110]. $\mathrm{Yu}$ found that the AP root ethanol extract could delay the occurrence of Alzheimer's disease by increasing serum superoxide dismutase (SOD) and reducing acetylcholine esterase (AChE) in brain tissue [111]. Osthol in AP could regulate Jak-SAKT, MAPK, PI3K, TGF- $\beta$, phosphatidylinositol, and other signaling pathways by participating in the metabolism of glycerol phospholipid, purine, niacin, and nicotinamide. Activation of the PI3K/Akt signaling pathway increases the survival rate of BM-NSCs and inhibits apoptosis to protect BM-NSCs injured by $\mathrm{H} 2 \mathrm{O} 2$ [112]. We speculated that after being absorbed into the body, osthol would undergo a series of metabolic reactions to form a lipid soluble metabolite, which would pass through the bloodbrain barrier and play a neuroprotective role. But, the metabolomics of osthol at the animal and cellular levels has not been studied. In the future research, the mechanism of action and metabolomics of monomer compounds which play an important role should be studied and analyzed.

By regulating the Notch signaling pathway, BM-NSCs can be promoted to express and differentiate into cholinergic neurons, which can play a role in Alzheimer's disease [114]. And, it played a neuroprotective role by promoting the expression of cyclic adenosine phosphate reactive element binding protein (CREB) and brain-derived neurotrophic 
factor (BDNF) and increasing the expression of $\mathrm{p}$-CREB and BDNF [114]. However, most of the above studies focused on the crude extract of AP, and there were few studies on the monomer compounds in AP. In the future, monomer compounds should be studied and analyzed, and their unknown mechanism of action in clinical treatment should be clarified.

7.4. Antibacterial Effect. It had been reported that AP decoction had significant inhibitory activity on tubercle bacillus and brucella [117]. And, the chloroform extract of Angelica polyclada Franch showed inhibition effect on $\mathrm{Ba}$ cillus subtilis and Escherichia coli, among which the inhibitory activity against Escherichia coli was stronger than that of Bacillus subtilis [34]. Zhang et al. used the growth rate method to test the inhibitory effect of the extracts from the petroleum ether layer of AP on the growth of 12 kinds of pathogenic bacteria mycelium. The experimental results showed that the extract had antibacterial effect on the tested bacteria, the experimental results showed that AP had different antibacterial effects on 12 tested fungi, and the inhibition rate of Rhizoctonia solani was the strongest, reaching $100 \%$, followed by Sclerotinia sclerotiorum and Phytophthora capsici, reaching over 80\%, while for Exserohilum turcicum and Magnaporthe oryzae, the inhibition rate was relatively low, and preliminarily identified pimpinellin was the main active component, with strong antibacterial activity [115]. Li et al. isolated and studied the antibacterial active components of the ethanol extract of AP and the results showed that isobergamolactone, sphondin, pimpinellin, and isopimpinellin were the main antibacterial substances, and the EC50 of isobergamolactone, pimpinellin, and isopimpinellin on citrus anthrax was $45 \mathrm{mg} / \mathrm{L}$, among which sphondin had the best inhibitory activity on citrus anthrax, and its EC50 was $41.5216 \mathrm{mg} / \mathrm{L}$ [116]. However, the absence of the positive control group and the blank control group and a lack of dose-dependent effects were two limitations of the studies.

7.5. Antioxidation Effects. Min et al. found that the antioxidant effect of AP was related to the polyphenol compounds contained in it. The study by Chen et al. had shown that the content of malondialdehyde (MDA) in the brain tissues of mice could be reduced by the decoction and alcohol extract of AP, and the effect of the AP alcohol extract was better than that of the water extract, which may be related to the more effective compound coumarin contained in the alcohol extract [96].. Studies by Hou et al. showed that the alcohol extract of AP could inhibit the concentration of MDA in the mouse plasma, and the inhibitory rate could reach $23.49 \%$. Zhang and Du found that AP could delay the occurrence of Alzheimer's disease by increasing the level of serum superoxide dismutase (SOD) [111]. And, Lu and Zhang showed that the ethanol extract of AP had significant antioxidant effect on five edible oils. However, the study was carried out at a single dose and lacked concentration dependence [122]. As a common TCM, AP has high safety and is widely distributed in various provinces of China. If further study is performed on its antioxidant activity, it is considered possible to make it a natural antioxidant.

7.6. Antitumor Effect. Zou et al. studied different concentrations of AP extracts at the cellular level and found that AP inhibited proliferation of human liver cancer cell lines (SMMC-7721) and human umbilical vein endothelial cells (HMVECs) in a dose-dependent manner, with an IC50 value of $1.59 \mathrm{mg} / \mathrm{ml}$. Under the inhibition of $20 \%$ of SMMC-7721, the inhibition of HMVECs was more than 50\%, showing that there was a significant dose difference between the two cell lines [96]. Lin et al. showed that psoralen, bergapten, xanthotoxin, umbelliferone, osthol, isoimperatorin, and other compounds in AP had antitumor effects, and their antitumor mechanisms may be related to inducing tumor cell apoptosis, inhibiting tumor cell DNA replication, inhibiting tumor cell multidrug resistance, and inhibiting tumor cell metastasis $[4,123]$. However, this was only the study on the antitumor mechanism of monomer compounds, not AP. And, few studies had conducted comparative studies of the antitumor effects of the crude AP extract and specific compounds in AP. In the future, scholars should also screen out the compounds whose antitumor effects of AP play a dominant role and strengthen their studies on cell and animal levels.

7.7. Others. The study by Dan et al. showed that chloroform, petroleum ether, and ethyl acetate extracts of AP had significant antigastric ulcer effects [98]. Psoralen-derived compounds, such as bergapten, xanthotoxin, and other compounds, could cause solar dermatitis in humans. Although some experiments had shown that AP has antigastric ulcer and sensitization effects, its mechanism had not been elucidated.

\section{Toxicity}

Bielu records "AP as sweet, lukewarm, and nontoxic." The 2015 edition of the Chinese Pharmacopoeia stipulates that the safe dose of AP is 3-10 g per day for adults. Studies have shown that AP contains xanthotoxin, bergapten, psoralen, and osthol, all of which have a certain degree of photosensitivity. Taking too much AP may cause a range of toxic effects. It was recorded in the Chinese Materia Medica (中华 本草) that the LD50 of intramuscular injection of xanthotoxin in rats was $160 \mathrm{mg} / \mathrm{kg}$ and the LD50 of bergapten was $945 \mathrm{mg} / \mathrm{kg}$. $400 \mathrm{mg} / \mathrm{kg}$ xanthotoxin could cause death and adrenal hemorrhage in guinea pigs. $200-300 \mathrm{mg} / \mathrm{kg}$ xanthotoxin could cause liver turbidity, fatty change, acute hemorrhagic necrosis, severe renal congestion, and hematuria. Continuous administration of $1-2 \mathrm{mg} / \mathrm{kg}$ xanthotoxin for 5 months could lead to liver necrosis. The LD50 of osthol intraperitoneally injected in mice was $16 \mathrm{mg} / \mathrm{kg}$ [25].

Another report was on two cases of AP poisoning: (1) a male, 9 years old, accidentally ate AP about $100 \mathrm{~g}$, and $1 \mathrm{~h}$ after eating, he began to vomit, was irritable and incoherent, had whole bodies convulsions, went into coma, and finally died; (2) a male, 7 years old, accidentally ate AP about $50 \mathrm{~g}$, 
and $1 \mathrm{~h}$ after eating, he began to vomit and had restlessness. Physical examination revealed the following: body temperature was $36.2^{\circ} \mathrm{C}$, pulse was 82 times $/$ min, blood pressure was $100 / 80$, dilated double pupils, slow response to light, arrhythmia, peripheral blood leukocytes were 20400, neutrophils were $83 \%$, and lymphocytes were $17 \%$ [124].

Kunming mice were used for the acute toxicity test, and the results showed that 10 minutes after oral administration of large dose $(15.80 \mathrm{~g} / \mathrm{kg})$, the mice developed symptoms of poisoning such as tail tip cyanosis, restlessness, and accelerated breathing, and some of the mice died due to respiratory failure, LD50 was $7.35 \pm 0.62 \mathrm{~g} / \mathrm{kg}$. Long-term toxicity tests were conducted on Wistar rats and hybrid dogs. The results showed that $10 \mathrm{~min}$ after oral administration of large dose $(825 \mathrm{mg} / \mathrm{kg})$, the rats developed symptoms of poisoning such as tail tip cyanosis, restlessness, and rapid breathing. However, the poisoning symptoms disappeared 30 days after oral administration. The growth rate of the high-dose group was slower than that of the control group $(P<0.01)$. Pathological examination revealed gastric flatulence and mucosal edema in some rats. Under a light microscope, hyaline bodies were observed in a few rats and dogs $(138 \mathrm{mg} / \mathrm{kg})$ in the high-dose group [125].

At present, there are few studies on the toxicity of AP, and future scholars should focus on the toxicity of AP. In order to ensure the safety and efficacy of AP, toxicity evaluation can provide guidance for clinical drug efficacy and patient safety [126].

\section{Conclusion and Prospect}

AP, as a famous TCM, with complex chemical components and extensive pharmacological effects, has been widely used in China for more than 2000 years. Scholars have done a lot of research on AP. So far, more than 120 coumarin compounds and 220 volatile oils compounds had been isolated from AP. It has also developed from the initial treatment of rheumatism alone to the modern prevention and treatment of tumor, cardiovascular and cerebrovascular diseases, and various arthritis diseases, which provides great help for its development and clinical application. However, there is still a lot of work to be done on the development and utilization of AP.

First, for the quality control of AP, the species of AP are complex, and the species of AP collected in different regions are not the same, and the identification of appearance and character alone is not perfect. Therefore, further research and demonstration are needed to identify authentic AP products, substitutes, and differences in their efficacy. The Chinese Pharmacopoeia takes the dry root of Angelica pubescens Maxim, f. biserrata Shan et Yuan as authentic AP product, which only takes the content of osthol and columbianadin as the quality control standard of AP, which lacks reliability because there is no research to prove that these two compounds are bioactive substances of AP. And, we should analyze the whole characteristics of AP to find out the most effective active ingredients and establish the quality control standard of AP on this basis.
Second, the processing of TCM has the effect of enhancing effect and reducing poison, which is widely used in the processing of TCM. However, there are no reports on processing AP in modern studies including phytochemistry, pharmacology, pharmacodynamics, and pharmaceutical analysis. Studies have shown that APR enters the kidney meridian and bladder meridian. It has a better pharmacological effect among TCMs which enters the kidney meridian after being baked with salt or wine. We speculate that AP can induce the decline of the body after salt roasting, which can better treat knee osteoarthritis and increase the analgesic function of the drug. And, AP after wine roasting can induce medicine to go to the top of the body, relieve headache better, and enhance the effect of blood circulation and activating collaterals. Therefore, it is necessary to study the processing products of AP, which can also fill the gap of AP processing.

Third, the research on the pharmacodynamic substances of AP is not thorough, and the mechanism, target, and pathway of the action of the active ingredients on the disease have not been scientifically clarified. Modern scholars can consider using network pharmacology, data mining, and virtual screening techniques to predict the chemical components and pharmacological activity related to the target, receptor, and pathway and verify through pharmacological experiments. In addition, new data mining methods can be proposed to screen herbs with similar effects, so as to reduce the development cost of new drugs by using natural drugs more effectively. And, most of the research studies focus on preliminary experiments in animals, and more in vitro studies at the cell level and more comprehensive clinical application are needed to further confirm its pharmacological mechanism. It is believed that with the innovation of the theories of phytochemistry, pharmacology, pharmacodynamics, etc., and the technical means of chromatographymass spectrometry, metabonomics, etc., a more scientific and reasonable quality control standard will be formulated, and the mechanism, target, and channel of its effective components will also be clarified, which will make up for the shortage of modern application of TCMs.

Fourth, in order to ensure safety and effectiveness, toxicity assessment can provide guidance for clinical efficacy and patient safety. At the same time, toxicity evaluation is also a prerequisite for the development of new drugs. However, there are few reports on the toxicity evaluation and the absorption, distribution, metabolism, and excretion of the main active components of AP in vivo. Future scholars should also study the toxicity and pharmacokinetic characteristics of the compounds in AP, so as to provide experimental basis for AP to become a new drug.

$\mathrm{AP}$, as a TCM, plays an important role in maintaining human health. We hope that this paper can provide more understanding of AP and provide guidance for its future research. We also firmly believe that with the continuous deepening of the research on AP, its application can be extended from the clinical medicine field to health products, cosmetics, and other fields, and occupy a place in the world market. 


\section{Data Availability}

All reported or analyzed data in this review are extracted from published articles.

\section{Conflicts of Interest}

The authors declare no conflicts of interest.

\section{Authors' Contributions}

Haixue Kuang, Qiuhong Wang, Bingyou Yang, and Hai Jiang conceived and designed the review; Ajiao Hou, Wenjing Man, Xinyue Guo, Song Wang, and Jiaxu Zhang searched the literature and downloaded the documents and made classification; Ajiao Hou wrote the paper; and Liu Yang checked the chemical structures and formula and contributed comments for version of the manuscript. All authors read and approved the final manuscript.

\section{Acknowledgments}

This work was financially supported by the National Natural Science Foundation of China (Grant nos. 81973604, 81803690, and 81703684), the Special Funds from the Central Finance to Support the Development of Local Universities, Postgraduate Funds for Heilongjiang University of Chinese Medicine (no. 2019yjscx013), the National Natural Science Foundation Matching Project (nos. 2018PT02 and 2017PT01), the Innovative Talents Funding of Heilongjiang University of Chinese Medicine (no. 2018RCD25), the Postdoctoral Initial Fund of Heilongjiang Province (UNPYSCT 2017219), the University Nursing Program for Young Scholars with Creative Talents in Heilongjiang Province (no. UNPYSCT-2017215), the Natural Science Foundation of Heilongjiang Province (no. H2015037), the Heilongjiang University of Chinese Medicine Doctoral Innovation Foundation (nos. 2014bs05 and 2013bs04), the Application Technology Research and Development Projects of Harbin Technology Bureau (no. 2014RFQXJ149), and the Heilongjiang Postdoctoral Scientific Research Developmental Fund (nos. LBH-Q16210 and LBH-Q17161).

\section{References}

[1] S. Q. Chen, H. Q. Zhang, C. Q. Yuan, Y. Q. Deng, and G. Y. Chen, "Studies ON the coumarins from the Chinese crude drugs chuandh (root $\mathrm{OF}$ angelica pubescens maxim. F. Biserrata shan et yuan) and zhedh (root OF a. Pubescens maxim.)," Acta Pharmaceutica Sinica, vol. 17, no. 5, pp. 392-394, 1982.

[2] National Pharmacopoeia Committee, The Pharmacopoeia of the People's Republic of China, China Medical Science and Technology Press, China, 2015.

[3] L. Y. Zhang and M. X. Liang, "Exploration and analysis on the potential function of Radix Angelicae Pubescentis," Zhong Hua Zhongyao Zazhi, vol. 33, no. 1, pp. 46-49, 2018.

[4] L. Lin, X. P. Qian, and B. R. Liu, "Research progress on the chemical constituents and the anti-tumor activity of
Angelica Pubescens," Journal of Modern Oncology, vol. 19, no. 2, pp. 373-376, 2011.

[5] X. L. Guo, X. M. Lin, J. Guo, and J. M. You, "The research status and prospect of radix angelicae pubescentis," Journal of Anhui Agricultural Sciences, vol. 42, no. 33, pp. 11673-11674, 2014.

[6] G. Zhou and B. H. Ma, "The development on the study of Radix Angelicae Pubescentis," China Modern Medicine, vol. 19, no. 16, pp. 15-16, 2012.

[7] Y. Zhu, F. Li, and Q. Y. Liu, "Research advances in the study of herbalism and pharmacology of traditional Chinese medicine of Radix Angelicae Pubescentis," Journal of Liaoning Economic Management Cadre Institute, vol. 1, pp. 68-69, 2010.

[8] W. Wang, Study on the Evaluation of Quality of Traditional Chinese Medicine Radix Angelicae Pubescentis, Liaoning University of Traditional Chinese Medicine, Shenyang, China, 2005.

[9] Y. T. Ma, "Studies on Radix Angelicae Pubescentis and Rhizoma Dioscoreae Nippomcae, " Northwest University, Kirkland, USA, 2005.

[10] J. H. Liu, S. X. Xu, and X. S. Yao, "Research advances in the study of chemical constituents and pharmacology of Radix Angelicae Pubescentis," Journal of Shenyang College of Pharmacy, vol. 11, no. 2, pp. 143-150, 1994.

[11] C. Pan, "The compatibility, identification, application and application in veterinary clinic of Radix Angelicae Pubescentis," Journal of Veterinary Medicine, vol. 9, p. 235, 2014.

[12] G. B. Gong and H. L. Wu, "Clinical application of common drug pairs of radix angelicae pubescentis," Modern Journal of Integrated Traditional Chinese and Western Medicine, vol. 14, no. 20, pp. 2653-2654, 2005.

[13] H. Shen, L. C. Mu, F. Wang, and L. M. Xie, "Efficacy of a pair of notopterygii rhizoma et radix-angelicae pubescentis radix in external use," Chinese Journal of Experimental Traditional Medical Formulae, vol. 24, no. 15, pp. 228-234, 2018.

[14] K. X. Duan, Y. W. Li, H. B. Liu, and B. L. Wang, "Study on Anti-inflammatory mechanism of couplet medicine of notopterygium incisum-angelica pubescens based on network pharmacology," China Pharmacy, vol. 30, no. 9, pp. 1241-1246, 2019.

[15] W. Cao and C. C. Li, "Herb textual research on and common pairs of doubleteeth pubescent Angelica root," Journal of Shaanxi University of Chinese Medicine, vol. 41, no. 1, pp. 127-130, 2018.

[16] X. F. Cao and L. Zhou, "HPLC determination of osthole in DHjisheng pills," Chinese Practical Medicine, vol. 5, no. 9, pp. 36-37, 2010.

[17] J. Wang, J. Q. Chen, L. H. Yin et al., "HPLC simultaneous determination of glycyrrhizic acid and osthole in Extract of DH Gegen Decoction," Chinese Journal of Pharmaceutical Analysis, vol. 30, no. 3, pp. 2052-2054, 2010.

[18] L. L. Huang, "The clinical application of Radix Angelicae Pubescentis," China's Naturopathy, vol. 11, no. 8, pp. 45-46, 2003.

[19] Y. Xiang, S. H. Yang, G. X. Yao, S. J. Fan, and Z. W. Luo, "The cultivation techniques and medicinal value of Radix Angelicae Pubescentis," Special Economic Animal and Plant, vol. 7, pp. 27-28, 2019.

[20] L. Y. Du, J. C. Su, Q. Guo et al., "A AP cultivation method," 2020.

[21] G. X. Liu, “A AP cultivation greenhouse,” 2019.

[22] J. Pan, "A method for cultivating high-quality AP," 2019. 
[23] W. Wu, "A AP cultivation method for high qand high yield," 2020.

[24] Nanjing University of Chinese Medicine, ZhongYaoDaCiDian", Shanghai Science and Technology Press, , Shanghai Science and Technology Press, China, 2nd edition.

[25] Editorial Board of National Administration of Traditional Chinese Medicine, The Chinese Materia Medica, Shanghai Science and Technology Press, China, 1999.

[26] P. Che, X. M. Ji, S. Liang, Y. Y. Zhang, and T. Han, “Antiinflammatory and analgesic effects and change of serum 5HTP and 5-HIAA by DH jisheng decoction on adjuvant arthritis rats," Chinese Journal of Experimental Traditional Medical Formulae, vol. 20, no. 19, pp. 170-173, 2014.

[27] Y. L. Tang and J. K. Wang, "A preliminary study on the pharmacological effect of DH decoction," Journal of Traditional Chinese Medicine, vol. 24, no. 5, pp. 56-57, 2001.

[28] M. Hong, "The treatment of 120 cases of wind-dampnesscold joint pain with Duhui-Xixin decoction," Hubei Journal of Traditional Chinese Medicine, vol. 22, no. 4, p. 34, 1999.

[29] Q. J. Wang and Y. Wang, "Clinical efficacy of needle warming moxibustion combined with congrongduhuosan in treating ankylosing spondylitis with deficiency of kidneyyang type," Chinese Journal of Experimental Traditional Medical Formulae, vol. 16, 2020.

[30] B. M. Qian, "A kind of AP internal injuries fever health tea," 2012.

[31] D. N. Chen, "A kind of beauty dispelling wet medicinal wine," 2018.

[32] T. M. Yang, “A beauty mask cream," 2017.

[33] I. Pfeifer, A. Murauer, and M. Ganzera, "Determination of coumarins in the roots of Angelica dahurica by supercritical fluid chromatography," Journal of Pharmaceutical and Biomedical Analysis, vol. 129, pp. 246-251, 2016.

[34] Z. X. Wang, Y. Q. Shen, Y. J. Chen, and X. S. Yao, "Study on the active components of angelicae pubescentis maxim," Journal of Shenyang College of Pharmacy, vol. 5, no. 3, pp. 183-188, 1988.

[35] J. X. Pan, L. K. Arison, B. Smith, and J. G. Q. Han, "Separation and identification of iso-angelinol, anpubesol and other coumarins from Angelica pubescens Maxim, f. biserrata Shan et Yuan," Acta Pharmaceutica Sinica, vol. 22, no. 5, pp. 380-384, 1987.

[36] C. D. Wang, B. L. Qiao, S. Zhu, and L. H. Ju, "Chemical composition study of Angelica potymaropka Maxim," Northwest Journal of Pharmacy, vol. 6, no. 1, pp. 15-16, 1991.

[37] H. Q. Zhang, C. Q. Yuan, and G. Y. Chen, "Study on the chemical composition of Angelica biserraia," Chinese Traditional and Herbal Drugs, vol. 23, no. 10, pp. 515-516, 1992.

[38] G. F. Rao, Q. Yang, F. Cai, Q. M. Liu, and H. D. Sun, "The chemical composition of a Heracleum hemsleyanum Diels," Journal of Yunnan College of Traditional Chinese Medicine, vol. 17, no. 3, pp. 4-6, 1994.

[39] C. Y. Zhang, B. G. Zhang, and X. W. Yang, "Studies on the chemical constituents of the root of Angelica Pubescens F. Biserrata," Pharmaceutical Journal of Chinese People's Liberation Army, vol. 23, no. 4, pp. 241-245, 2008.

[40] Z. J. Cai, F. J. Dan, F. Cheng, J. Z. Wang, and K. Zou, "Chemical constituents of antibacterial activity fraction of Angelica polymorpha," Journal of Chinese Medicinal Materials, vol. 31, no. 8, pp. 1160-1162, 2008.

[41] X. F. Ding, X. Feng, Y. F. Dong, X. Z. Zhao, Y. Chen, and M. Wang, "Studies on chemical constituents of the roots of Angelica pubescens," Journal of Chinese Medicinal Materials, vol. 31, no. 4, pp. 516-518, 2008.
[42] X. F. Ding, X. Feng, Y. F. Dong, X. Z. Zhao, Y. Chen, and Y. Q. Liang, "Study on the coumarin components of Radix Angelicae Pubescentis," Chinese Traditional Patent Medicine, vol. 31, no. 7, pp. 1102-1104, 2009.

[43] Y. Zhu, "Comparison of the Active Components in Radix Angelicae Pubescentis of Different Places of Origin", Liaoning University of Traditional Chinese Medicin, Shenyang, China, 2007.

[44] X. W. Yang, Q. M. Guo, C. Y. Zhang, and B. G. Zhang, "Further studies on the chemical constituents of the root of Angelica pubescens f. Biserrata," PLA Journal of Pharmacy, vol. 24, no. 5, pp. 389-392, 2008.

[45] M. Wan, Y. Zhang, Y. Yang et al., "Analysis of the chemical composition of Angelicae Pubescentis Radix by ultra-performance liquid chromatogDHhy and quadrupole time-offlight tandem mass spectrometry," Journal of Chinese Pharmaceutical Sciences, vol. 28, no. 3, pp. 145-159, 2019.

[46] J. P. Hou, "Research development on volatile oil from chuanxiong rhizome," Journal of Medicinal Plant Research, vol. 6, no. 12, 2012.

[47] S. Y. Jia and B. Wang, "Determination of volatile components of JiDuhuo(Angelica biserrata)by HS-GC-MS," Anhui Medical and Pharmaceutical Journal, vol. 18, no. 8, pp. 1429-1433, 2014.

[48] Z. Z. Wang, H. F. Tang, Z. W. Su et al., "Microscopical identification and chemical analysis of traditional Chinese medicine "Jiuyanduhuo"” Academic Journal of Second Military Medical University, vol. 18, no. 2, pp. 153-156, 1997.

[49] H. P. Yao and Y. B. He, "Analysis of the volatile components of DH (Angelica pubescens Maxim.f.biserrata) by gas chromatography-mass spectrometry and multivariate curve resolution," Guiding Journal of Traditional Chinese Medicine and Pharmacy, vol. 22, no. 15, pp. 54-57, 2016.

[50] X. X. Chen, C. Z. Han, and H. W. Yang, "GC-MS analysis of the essential oils from Angelica Pubescen s," Journal of Nanjing College of Pharmacy, vol. 17, no. 4, pp. 252-255, 1986.

[51] C. M. Zhou, C. Yao, and H. L. Sun, "Study on the chemical composition of volatile oil from Radix Angelicae Pubescentis," Journal of Chinese Medicinal Materials, vol. 13, no. 8, pp. 29-32, 1990.

[52] Y. Li, L. F. Wang, K. P. Liu, B. Yang, C. Du, and Y. Wang, "Investigation of transdermal effect and transdermal composition of volatile oil from notopterygium incisum, Angelica pubescens and pair of them by GC-MS," Chinese Journal of Experimental Traditional Medical Formulae, vol. 18, no. 18, pp. $46-51,2012$.

[53] Z. C. R. Dun, G. H. Zhu, Y. Cai, Y. K. Xiong, Z. H. Yan, and E. Yuan, "Determination of volatile components in Tibetan medicine Heracleum millefolium by HS-GC-MS," Chinese Traditional and Herbal Drugs, vol. 48, no. 11, pp. 2182-2188, 2017.

[54] L. X. Pu, T. J. Tang, X. H. Yuan, D. B. Hou, and B. Wang, "Study on essential oil constituents of aralia cordata thunb. from different habitats," Medicinal Plant, vol. 38, no. 17, pp. 8946-8948, 2010.

[55] Q. Q. Ai, B. X. Gao, Z. Q. Lan et al., "GC-MS analysis of volatile oil from Heracleum souliei radix," Journal of Chengdu University of Traditional Chinese Medicine, vol. 39, no. 1, pp. 15-17, 2016.

[56] X. Z. Xie, Y. L. Wang, X. J. Pan, and L. F. Huang, "Analysis of volatile oils components in radix angelecae pubescentis by GC-MS," Chinese Journal of Spectroscopy Laboratory, vol. 29, no. 1, pp. 317-319, 2012. 
[57] H. Chen, C. H. Zhang, and H. Yang, "A comparative study on supercritical $\mathrm{CO}_{2}$ extraction and microwave extraction of the volatile Oil in Heracleum hemsleyanum Diels," Lishizhen Medicine and Materia Medica Research, vol. 18, no. 11, pp. 2732-2734, 2007.

[58] X. P. Wang, J. X. Qiu, and S. Li, "Rapid GC-MS analysis of essential oils in radix angelicae biseratae," Journal of Yanbian University (Natural Science Edition), vol. 37, no. 2, pp. 128-131, 2011.

[59] Z. H. Hou and F. X. Zou, "Gas chromatogDHhy/mass spectrometry(GC-MS) analysis of the essential oil from Angelica pubescehs Maxim. by molecular distillation separation," Journal of Qilu University of Technology, vol. 29, no. 4, pp. 16-20, 2015.

[60] L. L. Huang, S. P. Xiong, Z. Zhou, M. X. Huang, and K. Q. Wang, "Comparative study on chemical components of the volatile oil from the root of Angelica pubescens in different habitats," Journal of Wuhan Botanical Research, vol. 20, no. 1, pp. 78-80, 2002.

[61] Z. X. Zhang and X. L. Yang, "Research on volatile oil obtained from heracleum moellendorffii with different methods," Journal of Shaanxi University of Science \& Technology, vol. 27, no. 2, pp. 65-68, 2009.

[62] B. X. Gao, J. J. Deng, J. Zheng, and X. M. Lu, "GC-MS analysis of volatile oil from Radix Heracleum Candicans," Pharmacy \& Clinics of Chinese Materia Medica, vol. 5, no. 5, pp. 9-10, 2014.

[63] C. Y. Zhang, B. G. Zhang, and X. W. Yang, "GC-MS analysis of essential oil from the radix of Heracleum hemsleyauum Diels," Research Information on Traditional Chinese Medicine, vol. 7, no. 12, pp. 9-12, 2005.

[64] X. T. Wang, X. Wang, Q. Cai, and Y. Q. Fu, "Comparisons of volatile oil and TLCS of radix angelicae pubescentis from sichuan and radix angelicae pubescentis from northeast," Liaoning Journal of Traditional Chinese Medicine, vol. 34, no. 5, pp. 638-639, 2007.

[65] X. W. Yang, Y. F. Liu, H. Y. Tao, Z. Yang, and S. Y. Xiao, "GC-MS analysis of essential oils from Radix Angelicae Pubescentis," Journal of Chinese Materia Medica, vol. 31, no. 8, pp. 663-666, 2006.

[66] M. Fang, J. Ivanisevic, H. P. Benton et al., "Thermal degradation of small molecules: a global metabolomic investigation," Analytical Chemistry, vol. 87, no. 21, pp. 10935-10941, 2015.

[67] D. L. Jones, "Organic acids in the rhizosphere: a critical review," Plant and Soil, vol. 205, no. 1, pp. 25-44, 1998.

[68] L. Yang, H. Jiang, A. Hou et al., "Simultaneous determination of thirteen Q-markers in raw and processed tussilago farfara L. By UPLC-QQQ-MS/MS coupled with chemometrics," Molecules, vol. 24, no. 3, 2019.

[69] L. Yang, H. Jiang, S. Wang et al., "Discovering the major antitussive, expectorant, and anti-inflammatory bioactive constituents in tussilago farfara $\mathrm{L}$. Based on the spectrum-effect relationship combined with chemometrics," Molecules, vol. 25, no. 3, 2020.

[70] L. Yang, H. Jiang, X. Xing et al., “A biosensor-based quantitative analysis system of major active ingredients in Lonicera japonica thunb: using UPLC-QDa and chemometric analysis," Molecules, vol. 24, no. 9, 2019.

[71] É.A. Parfenov and L. D. Smirnov, "Pharmacological potential of antioxidants derived from coumarin (Review)," Pharmaceutical Chemistry Journal, vol. 22, no. 12, pp. 884-892, 1988.
[72] A.-H. Ge, W.-F. Ma, C.-P. Wang et al., "Ultra high performance liquid chromatography with photodiode array detector and quadrupole time-of-flight tandem mass spectrometry coupled with discriminant analysis to evaluate Angelicae pubescentis radix from different regions," Journal of Separation Science, vol. 37, no. 18, pp. 2523-2534, 2014.

[73] J. Wang and X. G. Gong, "Advances in studies on antitumor and immune regulation of polysaccharides," Chinese Journal of Biochemical Pharmaceutics, vol. 6, no. 4, pp. 674-682, 2001.

[74] Z. L. Xu, Q. Wamg, M. Zhao, Q. Xu, C. S. Jia, and Y. Y. Shi, "Test on the immunity-moderating function of Dioscorea oppostita thunb. Polysaccharide," Lishizhen Medicine and Materia Medica Research, vol. 18, no. 5, pp. 1040-1041, 2007.

[75] A. S. Luo, Z. Chun, S. R. Ge et al., "Effect of dendrobium denneanum polysaccharide reducing blood gllucose in vivo," Chinese Journal of Applied \& Environmental Biology, vol. 12, no. 3, pp. 334-337, 2006.

[76] G. H. Zhou and G. P. Yu, "Effect study of auricularia polysaccharide on reducing blood lipid," Modern Food Science and Technology, vol. 21, no. 1, pp. 46-48, 2005.

[77] C. W. Sun and G. Z. Zhong, "Study on the antioxidant effects of Astragalus polysaccharide (APS)," Chinese Pharmacological Bulletin, vol. 12, no. 2, pp. 161-163, 1996.

[78] A. J. Hou, T. Y. Chen, S. P. Peng et al., "Study on anti-aging effect of poria cocos polysaccharide," Pharmacology and Clinics of Chinese Materia Medica, vol. 20, no. 3, pp. 10-11, 2004.

[79] Y. F. Yang, Y. B. Zhang, and X. W. Yang, "Simultaneous determination of 24 trace elements of Angelicae Pubescentis Radix by ICP-OES and ICP-MS methods based on the microwave digestion," Chinese Journal of Pharmaceutical Analysis, vol. 36, no. 11, pp. 2004-2008, 2016.

[80] X. Li and J. Yu, "Determination of total coumarin in Radix Angelicae Pubescentis," China Journal of Chinese Materia Medica, vol. 6, no. 9, pp. 543-545, 1991.

[81] Z. F. Sha, W. J. Sun, H. Gao, and M. L. Miao, "Determination of osthole and columbianetin acetate in Angelica pubescens by reversed phase HPLC," Acta Pharmaceutica Sinica, vol. 26, no. 10, pp. 798-800, 1991.

[82] Y. X. Chang, Z. W. Zhu, J. Li, Q. H. Zhang, and X. W. Qin, "Simultaneous determination of the seven major constituents for quality control of radix angelicae pubescentis by HPLC coupled with chemometrics methods," Journal of Inner Mongolia University, vol. 42, no. 2, pp. 215-223, 2011.

[83] Y. H. Shi, S. M. Zhao, R. Wang, H. S. Tan, G. X. Yu, and Z. T. Wang, "Simultaneous determination of osthole and columbianadin of Radix Angelicae Pubescentis by reversed phase high performance liquid chromatogDHhy," Chinese Pharmaceutical Journal, vol. 45, no. 16, pp. 1270-1273, 2010.

[84] Y. L. Li and M. H. Gao, "Columbianctim in Angelica Pubescens maxim. F.biserrata shan et yuan by RP-HPLC," Guide of China Medicine, vol. 8, no. 19, pp. 59-60, 2010.

[85] W. Y. Liu, F. Feng, C. X. Yu et al., "Qualitative and quantitative analysis of the main constituents of radix ilicis pubescentis by LC-coupled with DAD and ESI-MS detection," Natural Product Communications, vol. 5, no. 1, pp. 23-26, 2010.

[86] B. Wang, X. Liu, A. Zhou, M. Meng, and Q. Li, "Simultaneous analysis of coumarin derivatives in extracts of Radix Angelicae pubescentis (Duhuo) by HPLC-DAD-ESI-MSn technique," Analytical Methods, vol. 6, no. 19, pp. 7996-8002, 2014. 
[87] W. X. Yang, "Divide the light intensity of light method to measurese Radix Angelcae Pubesentis inside the flavonoid's research," Science \& Technology Information, vol. 26, p. 391, 2007.

[88] F. J. Dan, Z. J. Cai, J. H. Guo, J. R. Pan, and L. Wang, "Determination of total coumarins in Angelica Polymorpha maxim," Journal of China Three Gorges University, vol. 30, no. 6, pp. 91-93, 2008.

[89] L. Wang, H.-L. Wu, X.-L. Yin, Y. Hu, H.-W. Gu, and R.-Q. Yu, "Simultaneous determination of umbelliferone and scopoletin in Tibetan medicine Saussurea laniceps and traditional Chinese medicine Radix angelicae pubescentis using excitation-emission matrix fluorescence coupled with second-order calibration method," Spectrochimica Acta Part A: Molecular and Biomolecular Spectroscopy, vol. 170, pp. 104-110, 2017.

[90] L. Yang, A. J. Hou, Y. P. Sun, S. Wang, J. X. Zhang, and H. Jiang, "Screening and quantifying the quality markers of DuHuo by fingerprint modelling," Journal of Liquid Chromatography \& Related Technologies, 2020.

[91] M. Ding, Y. Bai, J. Li et al., "A diol-based-matrix solid-phase dispersion method for the simultaneous extraction and determination of 13 compounds from Angelicae Pubescentis Radix by ultra high-performance liquid chromatography," Frontiers in Pharmacology, vol. 10, 2019.

[92] L. Jin, Q.-H. Zhang, J. He, E.-W. Liu, X.-M. Gao, and Y.-X. Chang, "An improved LC-MS/MS method for simultaneous determination of the eleven bioactive constituents for quality control of radix angelicae pubescentis and its related preparations," Scientific World Journal, vol. 2015, Article ID 365093, 10 pages, 2015.

[93] A. J. Hou and L. Yang, "A strategy for qualitative and quantitative profiling of Angelicae Pubescentis Radix and detection of its analgesic and anti-inflammatory components by spectrum-effect relationship and multivariate statistical analysis,” Biomed. Chromatogr, p. e4910, 2020.

[94] X. Li, J. Wang, and L. Gao, "Anti-inflammatory and analgesic activity of R.A.P. (Radix angelicae pubescentis) ethanol extracts," African Journal of Traditional Complementary \& Alternative Medicines, vol. 10, no. 3, pp. 422-426, 2013.

[95] L. Fan, L. Li, and H. F. He, "Pharmacological studies on antiinflammatory and analgesic effects of Radix angelicae pubeascentis's volatile oil," Anhui Medical and Pharmaceutical Journal, vol. 13, no. 2, pp. 133-134, 2009.

[96] H. S. Jin, "An Experimental Study on the Mechanism of Delaying Brain Aging with Radix Angelicae Pubescentis and its Alcohol Extract," Liaoning University of Traditional Chinese Medicine, Shenyang, China, 2001.

[97] S. Y. Chen, "Experimental Study of the Effects of Radix Angelicae Pubescentis on Malondialdehyde and Mitochondrial DNA Deletions in Naturally Aged Mice," Liaoning University of Traditional Chinese Medicine, Shenyang, China, 2005.

[98] X. Zou, R. P. Wang, H. Dai, and Y. Hu, "Experimental study on the antiangiogenic effect of radix angelicae pubescentis," Journal of Nanjing TCM University, vol. 24, no. 3, pp. 194196, 2008.

[99] F. J. Dan, Z. J. Cai, L. B. Zhu, J. Z. Wang, and R. P. Zhang, "Studies of anti-gastric ulcer active fraction of Angelica polymorpha maxim," Journal of China Three Gorges University (Natural Sciences), vol. 30, no. 3, pp. 87-88, 2008.

[100] R. Li, C. Zhao, M. Yao, Y. Song, Y. Wu, and A. Wen, "Analgesic effect of coumarins from Radix angelicae pubescentis is mediated by inflammatory factors and TRPV1 in a spared nerve injury model of neuropathic pain," Journal of Ethnopharmacology, vol. 195, pp. 81-88, 2017.

[101] W. C. Sun, L. H. Yang, Y. Qiu, J. Ren, R. Huang, and J. Fu, "Identify nature $\mathrm{N}$-acylethanolamide-hydrolyzing acid amide (NAAA) inhibitor: effect of Angelicae Pubescentis Radix on anti-inflammation," Journal of Chinese Materia Medica, vol. 36, no. 22, pp. 3161-3166, 2011.

[102] X. M. Ma, "Study on Antirheumatic Drugs for Effective Parts of Radix Angelicae Pubescentis," Liaoning University of Traditional Chinese Medicine, Shenyang, China, 2001.

[103] M. Li, J. H. Wen, F. Y. Ni et al., "Anti-inflammatory activity of two new sesquiterpenoids from Radix Angelicae Pubescentis," Acts Pharmaceutics Sinica, vol. 54, no. 2, pp. 343 347, 2019.

[104] R. Z. Li, Q. Y. He, M. Qiao, R. J. Meng, L. P. Ge, and Y. Gu, "Study on the active components of Radix Angelicae Pubescentis against platelet aggregation and inhibition of experimental thrombosis," Journal of Beijing Medical University, vol. 19, no. 12, pp. 23-25, 1988.

[105] R. Z. Li, Q. Y. He, Q. B. Zhang, R. J. Meng, L. Y. Wang, and $\mathrm{Y}$. Gu, "Study of the active ingredient of $\gamma$-aminobutyric acid in radix angelicae pubescentis," Journal of Peking University (Health Sciences), vol. 21, no. 5, p. 376, 1989.

[106] W. L. Chen and Y. Lu, "Study on the promoting blood circulation to remove blood stasis action of Radix Angelicae Pubescentis ethanol extract," Inner Mongol Journal of Traditional Chinese Medicine, vol. 24, no. 57, pp. 6-7, 2001.

[107] Y. Pei, D. X. Li, and S. H. Sun, "Effects of Radix Angelicae Pubescentis and its alcohol extracts on apoptosis in brain tissue of naturally aged mice," Chinese Journal of Gerontology, vol. 25, no. 8, p. 959, 2005.

[108] Y. Pei, X. D. Ma, J. Yi, S. Li, S. Y. Wang, and J. Ma, "Effects of Radix Angelicae Pubescentis on antioxidant function and glutamate content in Parkinson's disease model rats," Chinese Journal of Gerontology, vol. 34, no. 5, pp. 1272-1274, 2014.

[109] Y.-F. Yang, W. Xu, W. Song, M. Ye, and X.-W. Yang, "Transport of twelve coumarins from angelicae pubescentis radix across a MDCK-pHaMDR cell monolayer-an in vitro model for blood-brain barrier permeability," Molecules, vol. 20, no. 7, pp. 11719-11732, 2015.

[110] H. Q. Li, D. X. Li, and S. H. Sun, "Changes of mtDNAdeficient respiratory chain enzyme complex and the mechanism of action of Radix Angelicae Pubescentis in aging mice," Chinese Archives of Traditional Chinese Medicine, vol. 24, no. 2, pp. 279-281, 2006.

[111] J. Zhang and W. B. Du, "Effects of Radix Angelicae Pubescentis on the p38MAPK signal transduction pathway in the brain of dementia rats," Chinese Journal of Gerontology, vol. 30, no. 11, pp. 1514-1515, 2010.

[112] Z. X. Yu, "Effects of Radix Angelicae Pubescentis ethanol extract on learning and memory ability and related enzymes in alzheimer's disease model mice," Chinese Community Physician, vol. 18, no. 12, p. 7, 2010.

[113] D. Zhao, X. D. Zhang, H. G. Hao et al., "Study on Neural Protective Function of DH Experimental Allergic Encephalomyelitis," Research and Practice on Chinese Medicines, vol. 27, no. 1, pp. 31-33, 2013.

[114] Y. H. Yan, "Effect and Mechanism of Osthole in Angelica Pubescens with NY-BM-SCs Transplantation in the Treatment of AD, " Liaoning University of Traditional Chinese Medicine, Shenyang, China, 2017.

[115] Y. H. Yan, S. H. Li, L. Kong et al., "Neuroprotective effects of total coumarins in angelica pubescens against $\mathrm{A} \beta$-induced 
neurons damage," Liaoning Journal of Traditional Chinese Medicine, vol. 43, no. 8, pp. 1714-1717, 2016.

[116] Z. X. Zhang, "Studies on antifungal effect and compounds of Heracleam moellendorffii," Journal of Xianyang Normal University, vol. 22, no. 2, pp. 33-34, 2007.

[117] J. B. Qiu, Q. Xu, and X. H. Jiang, "Effects of ethanol extract of Angelicae Pubescentis Radix on cyclooxygenase," China Medical Herald, vol. 8, no. 16, pp. 42-43, 2011.

[118] R. J. Meng, Y. Gu, L. P. Ge, R. Z. Li, and Q. Y. He, "Effects of alcohol extract (H6F4) of Radix Angelicae Pubescentis on platelet aggregation and experimental thrombus formation," Chinese Traditional and Herbal Drugs, vol. 19, no. 12, pp. 23-25, 1988.

[119] Y. Huang, H. W. Wu, and Y. P. Wang, "Effect of intestinal absorption solution of Angelica pubescens on the tension of rat thoracic Aortic," Joumal of Basic Chinese Medicine, vol. 23, no. 2, pp. 187-190, 2017.

[120] J. Hu, L. Lin, X. P. Qian et al., "Experimental study of Angelica Pubescens and osthole isolated from Angelica Pubescens inhibiting angiogenesis in vitro," Journal Modern Oncology, vol. 21, no. 9, pp. 1945-1949, 2013.

[121] Y. Pei, J. Ma, S. Li et al., "Effect of radix angelicae pubescentis and coumarins $\mathrm{CHOP}$ and Caspasel2 in substantia nigra of rats with on expressions of Parkinson's disease," Chinese Archives of Traditional Chinese Medicine, vol. 37, no. 11, pp. 2579-2582, 2019.

[122] M. M. Li, J. H. Hu, L. He et al., "Isolation and identification of active compound from Radix Angelicae Biseratae extracts against citrus fungus pathogens," Journal of Fruit Science, vol. 29, no. 5, pp. 900-904, 2012.

[123] Y. C. Lu and X. M. Zhang, "Study on antioxidant effects on grease about radix angelicae pubesentis," Journal of Qinghai Junior Teachers' College, no. 5, pp. 74-76, 2004.

[124] L. Yao, H. X. Feng, H. Huo, B. Y. Liu, and N. Yu, "Pharmacological research review of osthole from $\mathrm{DH}$," Chinese Archives of Traditional Chinese Medicine, vol. 30, no. 10, pp. 2221-2225, 2012.

[125] Z. H. Diao, "Two cases of radix angelicae pubescentis poisoning," Chinese Journal of Pediatrics, vol. 22, no. 5, p. 270, 1983.

[126] Z. H. Diao, “Two cases of duhuo poisoning," Chinese Journal of Pediatrics, vol. 22, no. 5, p. 270, 1984. 